OPEN ACCESS

Edited by:

Tatiana Matveeva,

Saint Petersburg State University,

Russia

Reviewed by:

Zonghua Wang,

Fujian Agriculture and Forestry

University, China

Jihong Jiang,

Jiangsu Normal Univeristy, China

*Correspondence:

Chuan-Chao Dai

daichuanchao@ninu.edu.cn

Specialty section:

This article was submitted to

Plant Microbe Interactions,

a section of the journal

Frontiers in Plant Science

Received: 25 November 2016 Accepted: 20 January 2017

Published: 06 February 2017

Citation:

Zhou J, Li X, Chen Y and Dai C-C

(2017) De novo Transcriptome Assembly of Phomopsis liquidambari

Provides Insights into Genes Associated with Different Lifestyles in Rice (Oryza sativa L.)

Front. Plant Sci. 8:121.

doi: 10.3389/fpls.2017.00121

\section{De novo Transcriptome Assembly of Phomopsis liquidambari Provides Insights into Genes Associated with Different Lifestyles in Rice (Oryza sativa L.)}

\author{
Jun Zhou, Xin Li, Yan Chen and Chuan-Chao Dai * \\ Jiangsu Key Laboratory for Microbes and Functional Genomics, Jiangsu Engineering and Technology Research Center for \\ Industrialization of Microbial Resources, College of Life Sciences, Nanjing Normal University, Nanjing, China
}

The mechanisms that trigger the switch from endophytic fungi to saprophytic fungi are largely unexplored. Broad host range Phomopsis liquidambari is established in endophytic and saprophytic systems with rice (Oryza sativa L.). Endophytic P. liquidambari promotes rice growth, increasing rice yield and improving the efficiency of nitrogen fertilizer. This species's saprophytic counterpart can decompose rice litterfall, promoting litter organic matter cycling and the release of nutrients and improving the soil microbial environment. Fluorescence microscopy, confocal laser scanning microscopy and quantitative PCR investigated the colonization dynamics and biomass of $P$. liquidambari in rice in vivo. P. liquidambari formed infection structures similar to phytopathogens with infected vascular tissues that systematically spread to acrial parts. However, different from pathogenic infection, P. liquidambari colonization exhibits space restriction and quantity restriction. Direct comparison of a fungal transcriptome under three different habitats provided a better understanding of lifestyle conversion during plant-fungi interactions. The isolated total RNA of Ck (pure culture), EP (endophytic culture) and FP (saprophytic culture) was subjected to Illumina transcriptome sequencing. To the best of our knowledge, this study is the first to investigate Phomopsis sp. using RNA-seq technology to obtain whole transcriptome information. A total of 27,401,258 raw reads were generated and 22,700 unigenes were annotated. Functional annotation indicated that carbohydrate metabolism and biosynthesis of secondary metabolites played important roles. There were 2522 differentially expressed genes (DEGs) between the saprophytic and endophytic lifestyles. Quantitative PCR analysis validated the DEGs of RNA-seq. Analysis of DEGs between saprophytic and endophytic lifestyles revealed that most genes from amino acids metabolism, carbohydrate metabolism, fatty acid biosynthesis, secondary metabolism and terpenoid and steroid biosynthesis were up-regulated in EP. Secondary metabolites of these pathways may affect fungal growth and development and contribute to signaling communication with the host. Most pathways of xenobiotic biodegradation and metabolism were upregulated in FP. Cytochrome P450s 
play diverse vital roles in endophytism and saprophytism, as their highly specialized functions are evolutionarily adapted to various ecological niches. These results help to characterize the relationship between fungi and plants, the diversity of fungi for ecological adaptations and the application prospects for fungi in sustainable agriculture.

Keywords: Phomopsis liquidambari, rice, endophyte, saprophyte, transcriptome, ecological adaptation

\section{INTRODUCTION}

In farmland ecosystems, intensive agriculture weakens the selfcycle capacity of soil nutrients, forcing farmers to devote effort to collecting and burning plant litter and applying fertilizer to farmland. This leads to the waste of natural organic resources, atmospheric and environmental pollution and soil quality deterioration, which are unfavorable for sustainable agriculture. Therefore, it is necessary to find an appropriate medium to decompose plant residues in farmland in order to increase soil available nutrients.

Endophytic fungi and saprophytic fungi usually play important ecological functions in living plant tissue and dead plant material. Many studies have investigated the relationship between endophytes and saprophytes and have hypothesized that endophytes become saprophytic after senescence of host tissues (Hyde et al., 2007). This may be due to modification of host tissues during senescence, allowing fungal hyphae to penetrate the epidermis and colonize the surface of the host. Promputtha et al. (2007) isolated common endophytes from Magnolia liliifera, Phomopsis, Guignardia, Fusarium, and Colletotrichum that have a high degree of sequence similarity and are phylogenetically relevant to the corresponding saprophyte. These results suggest that some endophytes might alter their ecological strategies and adopt a saprophytic lifestyle. Promputtha et al. (2010) also reported that nine endophytes, Phomopsis sp. 2, Phomopsis sp. 6, Phomopsis sp. 10, Guignardia mangiferae, Corynespora cassiicola, Leptosphaeria sp., Fusarium sp. 1, Colletotrichum gloeosporioides and Colletotrichum sp. 2 were morphologically similar and phylogenetically related to saprophytes. These endophytes and their saprobic counterparts produce the same degrading enzymes and a similar isoform of $\beta$-mannanase. Fungal succession is relevant to enzyme production patterns during leaf decomposition, and the occurrence of saprophytes is related to enzyme production from endophytes. This provides further convincing evidence that endophytes can change their lifestyle to become saprophytes.

Lipids are an important component of all living cells that offer a structural basis for cell membranes and fuel for metabolism and have a role in cell signaling. Membrane lipid synthesis is a prerequisite of symbiosis, and the performance of the

\footnotetext{
Abbreviations: P. liquidambari, Phomopsis liquidambari; GFP, green fluorescent protein; Ck, pure culture/control treatment; EP, endophytic culture/ fungal mycelium of the P. liquidambari callus culture; FP, saprophytic culture/ litterfallcultivated fungal mycelium of $P$. liquidambari; DEGs, differentially expressed genes; DGE, digital gene expression profiling; dai, days after inoculation; NR, non-redundant databases; KEGG, Kyoto Encyclopedia of Genes and Genomes; COG, Clusters of Orthologous Groups of proteins; GO, Gene Ontology; FDR, false discovery rate; CDS, Protein coding region.
}

membrane depends on lipid composition (Wewer et al., 2014). Fatty acids and modified fatty acids are important molecules for pathogen colonizing plants whose functions include signaling, energy sources and virulence factors (Uranga et al., 2016). The oxylipins are a vast diversified family of secondary metabolites derived from oxidation of unsaturated fatty acids or further conversion (Tsitsigiannis and Keller, 2007). In fungi, precursors of oxylipins are usually linoleic acid, oleic acid and $\alpha$-linolenic acid (Pohl and Kock, 2014). Fungal oxylipins can be used as secondary metabolites that participate in infection processes, biotrophy and necrotrophy (Oliw et al., 2016). Fungi produce a series of secondary metabolites and small molecules that may not be directly required for growth, but play important roles in signal transduction, development and organism interaction. The cytochrome P450 enzyme system is thought to play various functions in biosynthesis of secondary metabolites and participated in biodegradation of lignin and various xenobiotics (Martinez et al., 2009).

Though most endophytes depend on readily available compounds such as soluble sugar to grow, xylariaceous endophytes can degrade cellulose and lignin (Promputtha et al., 2010). Hence, endophytes that produce enzymes to decompose lignin and cellulose could decompose host tissue and persist as saprophytes following host senescence. Our research shows that the endophyte Phomopsis liquidambari B3 can establish a symbiotic relationship with rice (Oryza sativa L.), systematically colonizing roots and aerial parts (Yang et al., 2014b), which promotes the growth of rice, increasing yield and significantly reducing application of nitrogen fertilizer (Yang et al., 2014a, 2015; Siddikee et al., 2016). In the saprophytic phase, the fungus can decompose rice straw, promote litter organic matter cycling and the release of nutrients, improve soil microbial environments (Chen et al., 2013a) and secrete laccase, cellulase and polyphenol oxidase. Dai et al. (2010a) investigated the capability to form cavities on the straw surface and the condition for laccase production in P. liquidambari, suggested that endophytes can form a series of cavities on straw to decompose plant materials by producing laccase. In addition, $P$. liquidambari also secretes degradative enzymes for phenanthrene (Dai et al., 2010b), indole (Chen et al., 2013c; Wang et al., 2014), ferulic acid (Xie and Dai, 2015), 4-hydroxybenzoic acid (Chen et al., 2011) and phytoestrogen luteolin (Wang et al., 2015) among others. The fungus utilizes these compounds as the sole carbon source for growth. Chen et al. (2013c) shows that the degradation rate of indole in endophyte B3 over $120 \mathrm{~h}$ in a pure culture condition was $41.7 \%$. The exogenous addition of plant litter significantly increased the ratio of indole degradation within $60 \mathrm{~h}$ to $99.1 \%$, indicating the utility of litter-induced fungi to produce laccase and lignin 
peroxidase to non-specifically decompose nitrogen heterocyclic compounds.

However, comparisons of different types of plant-fungal interactions in the same plant species are limited because saprophytic systems and mutualistic systems are separated in various plants. Therefore, it will be valuable to perform experiments studying saprophytism and mutualism in a single plant species to directly compare endophytic and saprophytic plant-fungi interactions. Few studies have directly compared two different plant-microbe interactions in a single plant species. Furthermore, because only a small part of plant cells are colonized and it is difficult to accurately detect expression levels of fungal genes in colonized tissue, the detection of gene expression profiles and elucidation of interactional mechanisms during the endophytic lifestyle transition at different stages in associated host tissues remain poorly understood. Recently, "omics" approaches have been used to better understand endophyte-plant interactions (Kaul et al., 2016).

Rice is a representative gramineous plant, the staple food for approximately half the global population and a model material in agricultural microbiology research. P. liquidambari is a broad-spectrum endophyte that is typically used to study the switch from endophytism to saprophytism. We have established experimental systems to study the endophytic and saprophytic interaction of $P$. liquidambari B3 with a rice single host. Colonization dynamics and distribution in rice in vivo were monitored by green fluorescent protein (GFP)-tagged $P$. liquidambari. To discuss the differences in gene expression in $P$. liquidambari B3 interactions with rice under the two conditions of endophytism and saprophytism transcriptome sequencing technology and digital gene expression profiles were used. Endophytes are known to establish a symbiosis with the host through a series of regulatory mechanisms, as there is a sizable difference in performance between mutualistic fungi in the host and the common saprophyte. However, there is insufficient research into the switch from endophyte to saprophyte function in senescent plant litter. Clarification of this scientific problem has great significance for understanding the relationship between endophytes and plants and for documenting the diversity of endophytes.

\section{MATERIALS AND METHODS}

\section{Fungal Strain and Transformation}

P. liquidambari B3 was isolated from the inner bark of Bischofia polycarpa. It was stored on a potato dextrose agar slant $(200 \mathrm{~g}$ potato extract, $20 \mathrm{~g}$ glucose, $20 \mathrm{~g}$ ager per liter, $\mathrm{pH} 7.0)$ at $4^{\circ} \mathrm{C}$. The fungus was activated in potato dextrose broth $(200 \mathrm{~g}$ potato extract, $20 \mathrm{~g}$ glucose per liter, $\mathrm{pH} 7.0$ ) and cultured for $48 \mathrm{~h}$ at $28^{\circ} \mathrm{C}$ with a rotation speed of $180 \mathrm{rpm}$.

The transformation vector plasmid for filamentous fungi pCT74 expresses sGFP under the control of the ToxA promoter. It contains the hygromycin B resistance gene $h p h$, which encodes an aminoglycosidic antibiotic and is derived from Streptomyces hygroscopicus. This gene has been used for selection and maintenance of transformed prokaryotic and eukaryotic cells. Protoplast preparation and transformation were performed as described by Yang et al. (2014b) with some modifications.

\section{Inoculation, Co-culture and Microscopy}

The rice cultivar used in this study was "Wuyunjing 21 ". Rice seeds were dehusked and surface-sterilized in $75 \%$ ethanol for $15 \mathrm{~min}$, bleached in a $6 \%$ sodium hypochlorite solution $(6 \%$ available chlorine) for $10 \mathrm{~min}$, rinsed repeatedly in sterile distilled water, and planted in 1/2 Murashige \& Skoog (MS) $0.7 \%$ agarose medium supplemented with $30 \mathrm{mM}$ sucrose for 4 days. The seedlings were kept vertical at $25^{\circ} \mathrm{C}$ under a $16 \mathrm{~h}$ of light at $22^{\circ} \mathrm{C}$ and $8 \mathrm{~h}$ of dark. Seedlings of roughly the same size were transferred to $1 / 2 \mathrm{MS}$ in a square petri dish $(13 \mathrm{~cm}$ in width, $13 \mathrm{~cm}$ in length). Each plate of five plants inoculated with 7-mm GFP-B3 mycelial disks were placed near the plant roots on the medium. Potato dextrose agar disks of equal size were used as a control. All treatments were replicated five times. Rice shoots and roots were sampled and processed for microscopy.

An Axio Imager A1 fluorescence microscope (Zeiss, Jena, Germany) was used for observing the fungal structures as described previously by Yang et al. (2014b). Confocal laser scanning microscopy was performed using a Ti-E microscope with an A1 confocal system (Nikon, Tokyo, Japan) to monitor the infection process. GFP and FITC images of rice shoots and roots were captured simultaneously using $488 \mathrm{~nm}$ excitation with an argon laser and fluorescence detection at $543.5 \mathrm{~nm}$. Images were processed using Adobe Photoshop CS6 (Adobe, San Jose, CA, USA).

\section{Quantification of $P$. liquidambari Biomass in Rice Roots and Shoots by Quantitative PCR}

$P$. liquidambari-infected roots and shoots were harvested at $0,3,7,14,21$, and 28 dai (days after inoculation). The biomass of $P$. liquidambari in the infected plant tissue was quantified using quantitative PCR (qPCR) according to Yang et al. (2014b). DNA was extracted after grinding tissue powder with a Multisource Genomic DNA Miniprep Kit (Axygen). A primer set suitable for qPCR was designed based on a $P$. liquidambari-specific ITS locus (Bf1 and Br1) (Table S1). PCR amplified products were cloned into the $\mathrm{pMD}^{\circledR}$ 19-T vector (Takara, Otsu, Japan) and expressed in competent DH5acells. Positive clones were screened and plasmids were extracted using a SanPrep Column Plasmid Mini-Prep Kit. A dilution range of the plasmids from $1.3 \times 10^{2}$ to $1.3 \times 10^{8}$ copies was used to make a standard curve. In the $\mathrm{qPCR}$ reaction system $(20 \mu \mathrm{L})$, gDNA was mixed with $\mathrm{SYBR}^{\circledR}$ Green Master Mix (Vazyme, Nanjing, China), primers, and $\mathrm{ddH}_{2} \mathrm{O}$. The PCR procedure was as follows: $94^{\circ} \mathrm{C}(1 \mathrm{~min})$ for one cycle; $94^{\circ} \mathrm{C}$ (15 s), $60^{\circ} \mathrm{C}(45 \mathrm{~s})$, and $72^{\circ} \mathrm{C}(30 \mathrm{~s})$ for 45 cycles; melting curve analysis from 72 to $60^{\circ} \mathrm{C}$ in $0.5^{\circ} \mathrm{C}$ decrements. The amplification of a single PCR product was validated using $1.5 \%$ gel electrophoresis. 


\section{Endophytic and Saprophytic Systems of $P$. liquidambari Interact with Rice}

Activated P. liquidambari was filtered with 8-layer gauze. Fungal mycelia were cleaned three times with sterile deionized water and $0.10 \mathrm{~g}$ mycelia ( $0.01 \mathrm{~g}$ dry biomass) were weighed and used for callus inoculation. In addition, $0.30 \mathrm{~g}$ mycelia $(0.03 \mathrm{~g}$ dry biomass) were weighed and inoculated in $20 \mathrm{~mL}$ sterile water as a fungus seed solution for litterfall inoculation; $0.30 \mathrm{~g}$ mycelia were weighed and inoculated in $1 \times \mathrm{NB}$ solid medium for 3 days and regarded as a control treatment $(\mathrm{Ck})$.

The endophytic lifestyle was studied using tissues cultures of host rice in dual culture in vitro based on previous research (Sieber et al., 1990; Peters and Schulz, 1998; Nawrot-Chorabik et al., 2016). Inoculated rice seeds at the surface were sterilized in callus solid medium $\left(1 \times \mathrm{NB}\right.$ solid medium, $2 \mathrm{mg} \mathrm{L}^{-1} 2,4-\mathrm{D}$, $30 \mathrm{~g} \mathrm{~L}^{-1}$ sucrose, $10 \mathrm{~g} \mathrm{~L}^{-1}$ agar, $\mathrm{pH} 7$ ) and cultivated for 20 days at $28^{\circ} \mathrm{C}$. The callus was stripped from rice seeds at germination and a yellow soft callus appeared at the base of medium. This was placed on new callus solid medium and cultivated for 50 days under $28^{\circ} \mathrm{C}$. When a callus formed (Figure S1), P. liquidambari mycelium was inoculated on top of the callus with tweezers and cultivated for 3 days at $28^{\circ} \mathrm{C}$. At this stage, the fungus was checked to ensure that it grew on the surface of the callus but did not contact the medium. The mycelium was stripped from the surface of the callus with delicate tweezers, and this sample was used as the fungal mycelium of the P. liquidambari callus culture (EP).

The saprophytic system was derived using the culture fungus method of Chen et al. (2013a) with litter. Collected rice litterfall completely withered from the ground of the rice experimental plot. The moisture content of rice litterfall was $7.6 \%$. The litterfall surface was washed with sterile deionized water and cut into $1 \mathrm{~cm} \times 1 \mathrm{~mm}$ segments. Weighed 0.5 -g litterfall samples were added to a $250-\mathrm{mL}$ triangular flask with $100 \mathrm{~mL} 1 \times \mathrm{NB}$ liquid medium ( $\mathrm{pH} 5.5)$ and sterilized for $20 \mathrm{~min}$ at $121^{\circ} \mathrm{C}$. A $2-\mathrm{mL}$ sample of $P$. liquidambari was inoculated into the seed solution and cultivated for 3 days at $28^{\circ} \mathrm{C}$ and $160 \mathrm{rpm}$. The mycelium pellet was removed from the liquid medium with tweezers and washed clean. This sample was used as a litterfall-cultivated fungal mycelium of $P$. liquidambari (FP).

\section{RNA Extraction}

Total RNA was isolated from Ck, EP and FP using a Fungal RNA extraction kit (E.Z.N.A. Total RNA Kit I, OMEGA, USA) and treated with DNase I. The quality and concentration of extracted RNA were examined using agarose gel electrophoresis and a spectrophotometer (OneDrop ${ }^{\mathrm{TM}} \mathrm{OD}-2000+$, China), and eligible groups were used for Illumina sequencing.

\section{cDNA Library Construction and Sequencing}

The mRNA of the total RNA was purified using magnetic Oligo (dT) beads. This mRNA was mixed with the fragmentation buffer, the mRNA was fragmented into short fragments. The cDNA was synthesized using mRNA fragments as templates. Short fragments were purified and resolved with EB buffer for sticky-end preparation and single nucleotide A addition. Subsequently, the short fragments were connected with adapters, and suitable fragments were selected as templates for PCR amplification. Quantification and qualification of the sample library was performed using an ABI StepOnePlus Real-Time PCR System and an Agilent 2100 Bioanalyzer. The library was sequenced using Illumina HiSeq ${ }^{\mathrm{TM}} 2000$.

\section{Sequence Annotation}

Image data output from Illumina sequencing was transformed by base calling into raw reads. Clean reads were obtained by removing dirty reads that contained adapters or unknown or low quality bases. Transcriptome de novo assembly was carried out with Trinty (Grabherr et al., 2011) and a k-mer library was constructed. The highest frequency k-mer was selected to assemble contigs and then mapped with clean reads. Paired-end reads were used to fill gaps in the scaffolds to assemble contigs to unigenes. Non-redundant unigenes were acquired by further processing of sequence splicing and removal of redundancy. Allunigenes were generated after gene family clustering. Unigene sequences were aligned with blastx $(e<0.00001)$ to protein databases including non-redundant databases (NR), Swiss-Prot, the Kyoto Encyclopedia of Genes and Genomes (KEGG) and the Clusters of Orthologous Groups of proteins (COG), and aligned by blastn $(e<0.00001)$ to the nucleotide databases nt. Gene Ontology (GO) functional annotation was achieved using NR annotation by Blast2GO (Conesa et al., 2005). GO functional classification was achieved using WEGO software (Ye et al., 2006).

\section{Identification of Differentially Expressed Genes}

The FPKM method was used to calculating unigene expression (Mortazavi et al., 2008). An algorithm to identify differentially expressed genes (DEGs) between the two samples was used according to the method of Audic and Claverie (1997). In our analysis, the genes with false discovery rates $(\mathrm{FDR}) \leq 0.001$ and ratios larger than 2 were regarded as significant DEGs. We mapped all DEGs to terms in the GO database and KEGG database for enrichment analysis.

\section{Quantitative Real-Time PCR Analysis}

To validate the DEGs obtained by Solexa RNA-seq, 20 genes (Table S1) were subjected to quantitative real-time PCR analysis using an ABI PRISM 7500 Real-time PCR System (Applied Biosystems). P. liquidambari $\beta$-actin (Table S1) was used as the endogenous control. cDNA synthesis was carried out using the same RNA samples as those used for digital gene expression profiling (DGE) experiments. The corresponding primers were designed using Primer Premier 6.0 and listed in Table S1. The reaction mixture $(20 \mu \mathrm{l})$ contained $10 \mu \mathrm{l}$ of SYBR ${ }^{\circledR}$ Green Master Mix (Vazyme, Nanjing, China), $0.4 \mu \mathrm{M}$ of forward and reverse primers, and $0.2 \mu \mathrm{l}$ of cDNA template. The amplification programs were performed with the following parameters: $95^{\circ} \mathrm{C}$ for $30 \mathrm{~s} ; 95^{\circ} \mathrm{C}$ for $5 \mathrm{~s}, 60^{\circ} \mathrm{C}$ for $40 \mathrm{~s}$ (40 cycles) and followed by melting curve analysis from 60 to $95^{\circ} \mathrm{C}$ in $0.5^{\circ} \mathrm{C}$ increments. Each reaction was run in triplicate, including a negative control. 
The relative expression levels of genes were calculated using the $2^{-\Delta \Delta C T}$ method.

\section{RESULTS}

\section{Infection and Colonization Process of Rice Plants Systemically by $P$. liquidambari}

To visualize the infection process of $P$. liquidambari in planta, we first obtained transgenic fungal strains by constitutively expressing cytoplasmic GFP (GFP-B3). In the early stage of infection (1-3 dai), P. liquidambari hyphae were only distributed on the root surface, especially the root-hair zone, infected roothairs and infected epidermis (Figures 1A-C). A large number of runner hypha interweaved together to form a hyphal network (Figures 1D-F and Video S1). Runner hypha were distributed in the low-lying area between the cells of root epidermal layers, growing along the longitudinal axis direction of the root and invading cells using a penetration peg (Figure 1G). In the middle stage of infection (4-15 dai), hyphae beginning intracellular and intercellular infection spread from epidermal layers to the cortex and finally to the endodermis (Figures $\mathbf{1 H}-\mathbf{J}$ ). In the epidermis and cortex, hyphae can undergo intracellular and intercellular growth along the direction parallel to the root spindle spread from one cell to another, branching in the intercellular space, and then continue to grow (Figures $1 \mathbf{K}-\mathbf{M}$ ). When it penetrated the cell wall, hyphae appeared with neck-like constrictions (Figure 1N). Parts of strong hypha entered the center of the root spindle and then penetrated the vascular bundle into the acrial part (Figures 1O-R). In late-stage colonization ( $>15$ dai), which is associated with programmed cell death, the vast majority of epidermal cells and parts of outer cortex cells were crowded with mycelium and sclerotium (Figures 1S,T). Colonization of $P$. liquidambari was still observed in the senescence root $(>50$ dai) (Figures 1U-W).

\section{Quantification of $P$. liquidambari Biomass in Rice Tissues}

The concentration of $P$. liquidambari within plantlets is expressed as the number of $P$. liquidambari-specific ITS copies per ng total (plantlet + fungal) genomic DNA in the qPCR analysis. The concentration of endophytes in roots was always higher than in shoots from 0 to 28 dai. In roots, a significant increase from 0 to 7 dai was followed by a moderate decrease. A a moderate increase in shoots occurred from 0 to 21 dai and then reached a steady state (Figure 2 ).

\section{Illumina RNA-Sequencing and Read Assembly}

To identify DEGs related to lifestyle, we mixed total RNA extracted from Ck, FP and EP of P. liquidambari hyphae equally for transcriptome sequencing. A total of 27,401,258 raw reads were generated. After filtering, 26,109,074 clean reads were obtained, for a total of 2,349,816,660 bp clean nucleotides. The Q20 percentage was $96.47 \%$ and the GC percentage was $56.45 \%$. After editing and quality checking, 26 million $90 \mathrm{bp}$ clean reads were assembled into 51,120 contigs with a mean length of $487 \mathrm{bp}$. The N50 of contigs was $1240 \mathrm{bp}$, where larger numbers are better for the quality of assembly. Using pairedend joining and gap filling, the contigs were further assembled into 32,424 unigenes with a mean length of $945 \mathrm{bp}$, including 7946 distinct clusters and 24,478 distinct singletons. The N50 of unigenes was $1574 \mathrm{bp}$, indicating that the assembly results were desirable (Table 1). The assembled sequence length is one evaluative criteria of assembly quality. The size distribution of the contigs and unigenes are shown in Figure 3. By comparing the length distribution proportion of contigs and unigenes, we found that contigs from 100 to $200 \mathrm{bp}$ accounted for $50.62 \%$, greater than 500 bp that accounted for $22.65 \%$ (Table S2). However, the length of unigenes obtained from further assembly, of which of $100-500$ bp accounted for just $46.48 \%$, were all greater than 200 bp and the proportion greater than 1000 bp was over $31.51 \%$ (Table S3), indicating that the assembly quality of unigenes that assembled from contigs was high.

\section{Functional Annotation of Predicted Proteins}

We matched unigene sequences against NR, NT, Swiss-Prot, KEGG, GO and COG databases using blastx $\left(E<10^{-5}\right)$. Of these, 22,700 unigenes (70.0\% of total) were annotated, and most could be annotated to protein functional information in the NR database. A total of 22,382 unigenes were annotated to NR database (Table S4). We have analyzed the E-value distribution (Figure 4A), similarity distribution (Figure 4B) and species distribution (Figure 4C) of the NR annotation. The $E$-value distribution showed that $64.8 \%$ of the mapped sequences displayed a high level of homology $\left(E<10^{-30}\right) ; 51.6 \%$ of the mapped sequences displayed a higher level of homology $(E<$ $\left.10^{-45}\right)$. The similarity distribution has a comparable pattern, with $50.5 \%$ of sequences having similarity higher than 60 and $12.5 \%$ of the sequences having similarity higher than $80 \%$. For species distribution, $8.2 \%$ of the distinct sequences had top matches with sequences from Colletotrichum higginsianum, followed by the Glomerella graminicola M1.001 (8.0\%), Gaeumannomyces graminis var. tritici R3-111a-1 (7.8\%), Thielavia terrestris NRRL 8126 (7.7\%), Magnaporthe oryzae 70-15 (6.3\%), Myceliophthora thermophila ATCC 42464 (5.7\%), Nectria haematococca mpVI $77-13-4(4.4 \%)$ and $48.1 \%$ of the unigene sequences matched to the seven species.

\section{GO and COG Classification}

GO functional annotation was obtained according to NR annotation information. GO assignments were used to classify the functions of the predicted $P$. liquidambari unigenes. A total of 10,209 unigenes were assigned to 50 functional groups in each of the three main categories according to sequence homology (Figure 5 and Table S5). In the cellular component, the majority was "cell," "cell part," "membrane," "organelle" and "membrane part" unigenes associated with cell membranes and organelles. In the section for molecular function, the dominant functions were "catalytic activity," "binding," "transporter activity" and "structural molecule activity." In the section for biological processes, unigenes were mainly involved in metabolic processes 

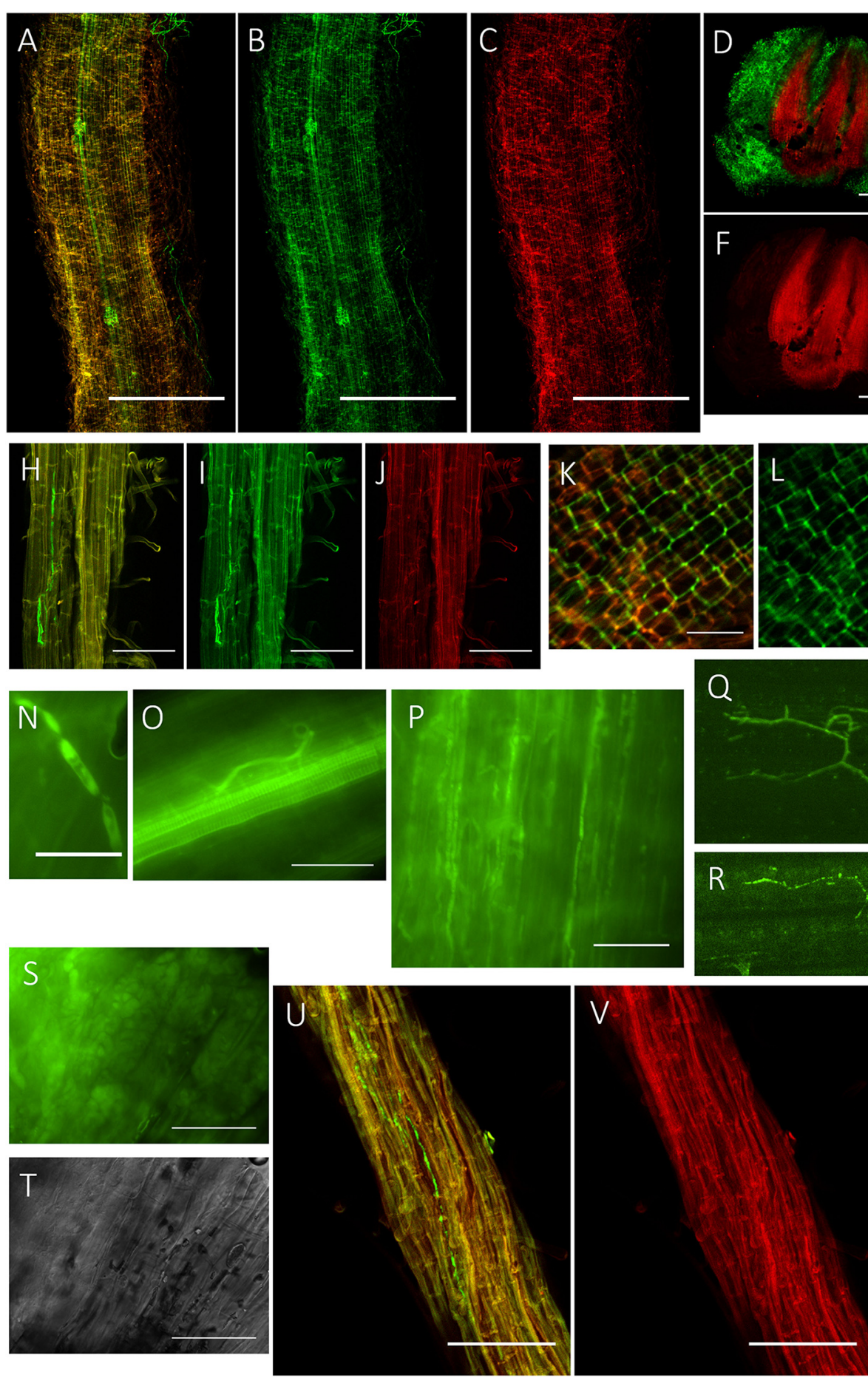

F

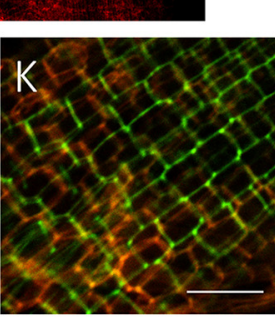

Q
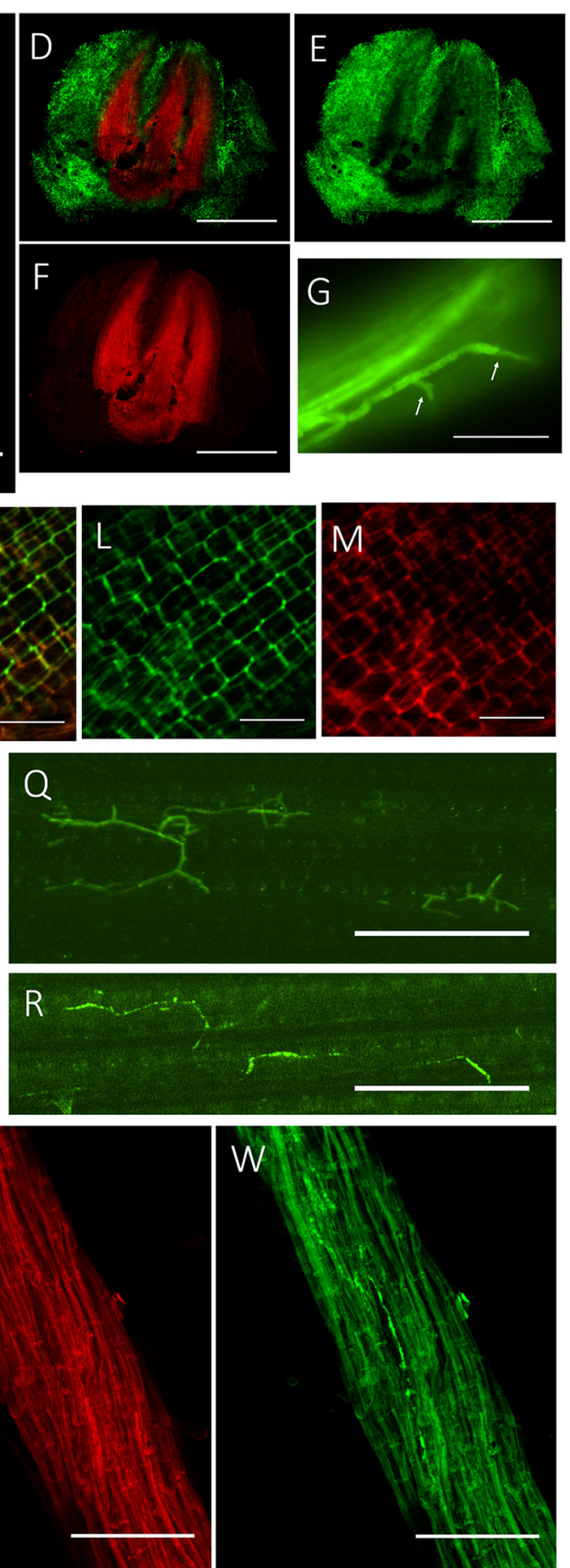

FIGURE 1 | Colonization pattern of $\boldsymbol{P}$. liquidambari in rice roots and shoots. (A-C) Distribution of GFP- P. liquidambari hyphae on the root surface and infection of root-hair; 1-3 dai. (A) Overlay channel. (B) Green fluorescence channel. (C) Red fluorescence channel. Bar, $100 \mu \mathrm{m}$. (D-F) Cross-section of root tip, runnner hypha interweaved to form hyphal network. (D) Overlay channel. (E) Green fluorescence channel. (F) Red fluorescence channel. Bars, 100 um. (G) Penetration peg (arrow). Bar, $25 \mu \mathrm{m}$. (H-J) Intracellular and intercellular growth of hypha along the direction parallel to the root spindle spread from one cell to another; 4-15 dai. Bar, $25 \mu \mathrm{m}$. (H) Overlay channel. (I) Green fluorescence channel. (J) Red fluorescence channel. (K-M) Hyphae branching in the intercellular space. (K) Overlay channel. (L) Green fluorescence channel. (M) Red fluorescence channel. Bar, $50 \mu \mathrm{m}$. (N) Neck-like constriction, Bar, $10 \mu \mathrm{m}$. (O) Penetration of the center of the root spindle. Bar, 25

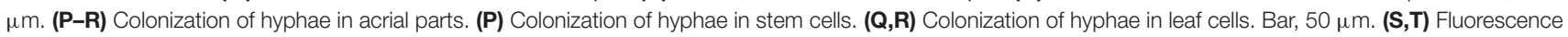
microscopy of root cells were crowded with mycelium and sclerotium (>15 dai). (S) DIC channel. (T) bright field channel. Bar, $25 \mu \mathrm{m}$. (U,V) Colonization of hyphae in senescence rice root (>50 dai). (U) Overlay channel. (V) Red fluorescence channel. (W) Green fluorescence channel. Bar, $50 \mu \mathrm{m}$. 


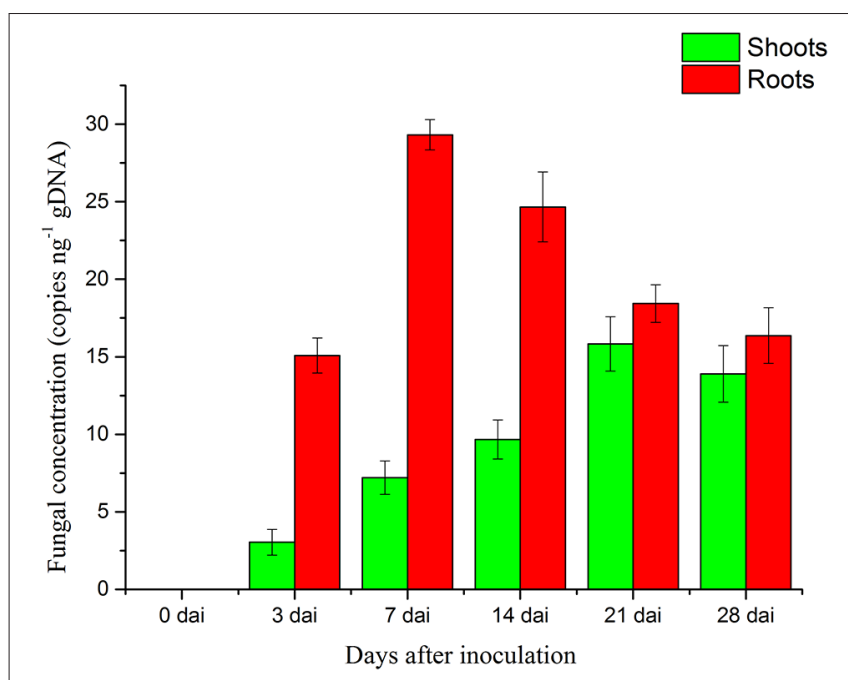

FIGURE 2 | Concentration of $\boldsymbol{P}$. liquidambari in infected rice tissue at various time points $(\mathbf{0}, \mathbf{3}, \mathbf{7}, \mathbf{1 4}, \mathbf{2 1}, \mathbf{2 8}$ dai). Values are means $\pm S D$ from three biological replicates.

TABLE 1 | Statistics of $P$. liquidambari transcriptome.

\begin{tabular}{ll}
\hline Total raw reads & $27,401,258$ \\
Total clean reads & $26,109,074$ \\
Total clean nucleotides (nt) & $2,349,816,660$ \\
Q20 percentage & $96.47 \%$ \\
N percentage & $0.01 \%$ \\
GC percentage & $56.45 \%$ \\
Total number of contigs & 51,120 \\
Total length of contigs (nt) & $24,882,464$ \\
Mean length of contigs (nt) & 487 \\
N50 of contigs & 1240 \\
Total number of unigenes & 32,424 \\
Total length of unigenes (nt) & $30,646,255$ \\
Mean length of unigenes (nt) & 945 \\
N50 of unigenes & 1574 \\
Distinct clusters & 7,946 \\
Distinct singletons & 24,478 \\
\hline
\end{tabular}

and cellular processes, in agreement with the results of cellular components and molecular function.

In total, 10,327 unigenes have a COG classification based on sequence homology. Among the 25 COG categories (Figure 6), "general function prediction only" (3698) was the largest group, followed by "carbohydrate transport and metabolism" (1998), "transcription" (1982), and "translation, ribosomal structure and biogenesis" (1887). The groups for "nuclear structure" (5), "extracellular structure" (25) and "RNA processing and modification" (74) were smallest.

\section{KEGG Analysis}

A total of 22,700 annotated unigenes of P. liquidambari were blasted to the KEGG database and annotated further. In all, 14,791 sequences were found to be involved in 108 signal pathways. The number of sequences ranged from 4 to 4785 (Table S6). The first 20 pathways with the greatest number of sequences are indicated in Table 2, and the pathways that were most represented were metabolic pathways (4785) and biosynthesis of secondary metabolites (2154). These annotations provided important clues for further studying the specific development, function and pathways of $P$. liquidambari. The top 10 metabolic pathways were as follows: starch and sucrose metabolism (1481), amino sugar and nucleotide sugar metabolism (814), purine metabolism (630), pyrimidine metabolism (408), lysine degradation (365), tyrosine metabolism (329), glycolysis/gluconeogenesis (276), fructose and mannose metabolism (249), butanoate metabolism (236) and tryptophan metabolism (226). We believe that genes in carbohydrate metabolism and biosynthesis of secondary metabolites play significant roles in $P$. liquidambari endophytism and saprophytism.

\section{Protein Coding Region (CDS) Prediction}

In total, 22,300 and 1551 unigenes were predicted by BLASTx and ESTScan, respectively. The histogram seen in Figure S2 shows the length distribution of CDS predicted by BLAST and ESTScan. In general, as sequence length increased, the number of CDS was gradually reduced. This is consistent with unigene assembly results.

\section{Analysis of Differentially Expressed Genes}

To detect the DEGs between EP and FP, we screened differentially expressed tags between samples according to the method described by Audic and Claverie (1997). As shown in Figure 7, there were 2869 genes that were differentially expressed between $\mathrm{Ck}$ and FP. Among these genes, 1502 were up-regulated and 1367 were down-regulated in response to the FP switch. There were 2277 genes differentially expressed between Ck and EP. Among these genes, 1382 genes were up-regulated and 895 were downregulated in response to the EP switch. There were 2522 genes differentially expressed between FP and EP. Among these genes, 1415 genes were up-regulated and 1107 were down-regulated in response to the switch between EP and FP. There were 491 genes co-expressed among the three expression patterns (Figure 7). DEGs were further categorized into different functional groups by GO and KEGG pathway enrichment analysis. Compared with $\mathrm{Ck}$, "starch and sucrose metabolism" and "amino sugar and nucleotide sugar metabolism" were the most enriched pathways in FP, and "butanoate metabolism" was the most enriched pathway in EP. Compared with FP, the "ribosome" group was the most enriched pathway in EP $(P<0.05)$ (Table S7).

\section{Validation of RNA-seq Data by qPCR}

To validate the DEGs obtained by Solexa RNA-seq, we further performed quantitative real-time PCR analysis on 20 representative genes involved in the three lifestyles (Figure 8). We found that fold-change values of most DEGs using realtime qRT-PCR exhibited trends similar to RNA-Seq samples. Differential expression was observed for all candidate genes, suggesting that they are involved in regulatory networks that are active during the three environmental conditions. Only 


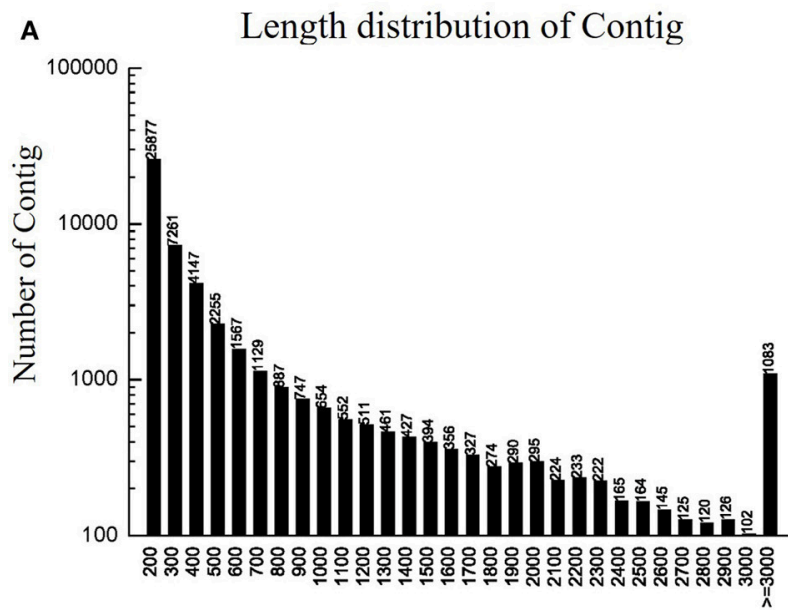

Sequence size (nt)

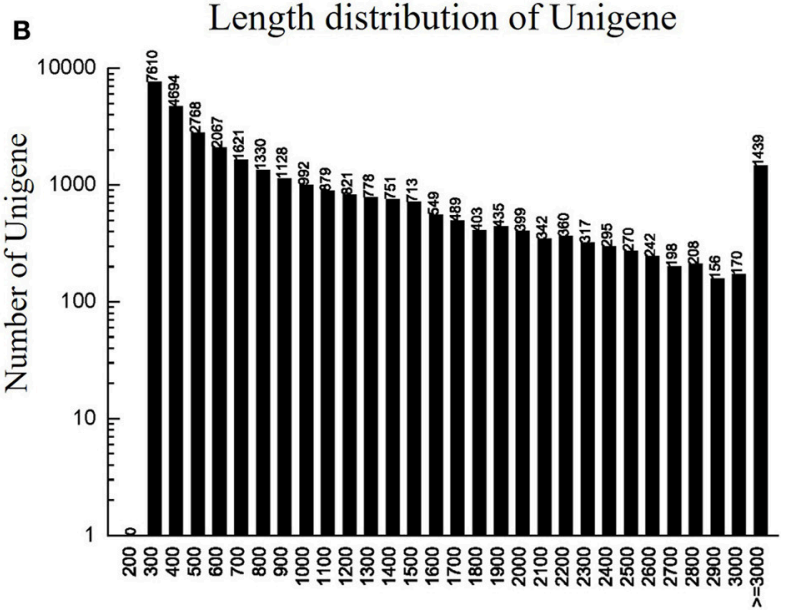

Sequence size (nt)

FIGURE 3 | Length distribution of contigs and unigenes. (A) Length distribution of contigs. Horizontal coordinate is contig length and vertical coordinate is contig number. (B) Length distribution of unigenes. Horizontal coordinate is unigene length and vertical coordinate is number of unigenes.

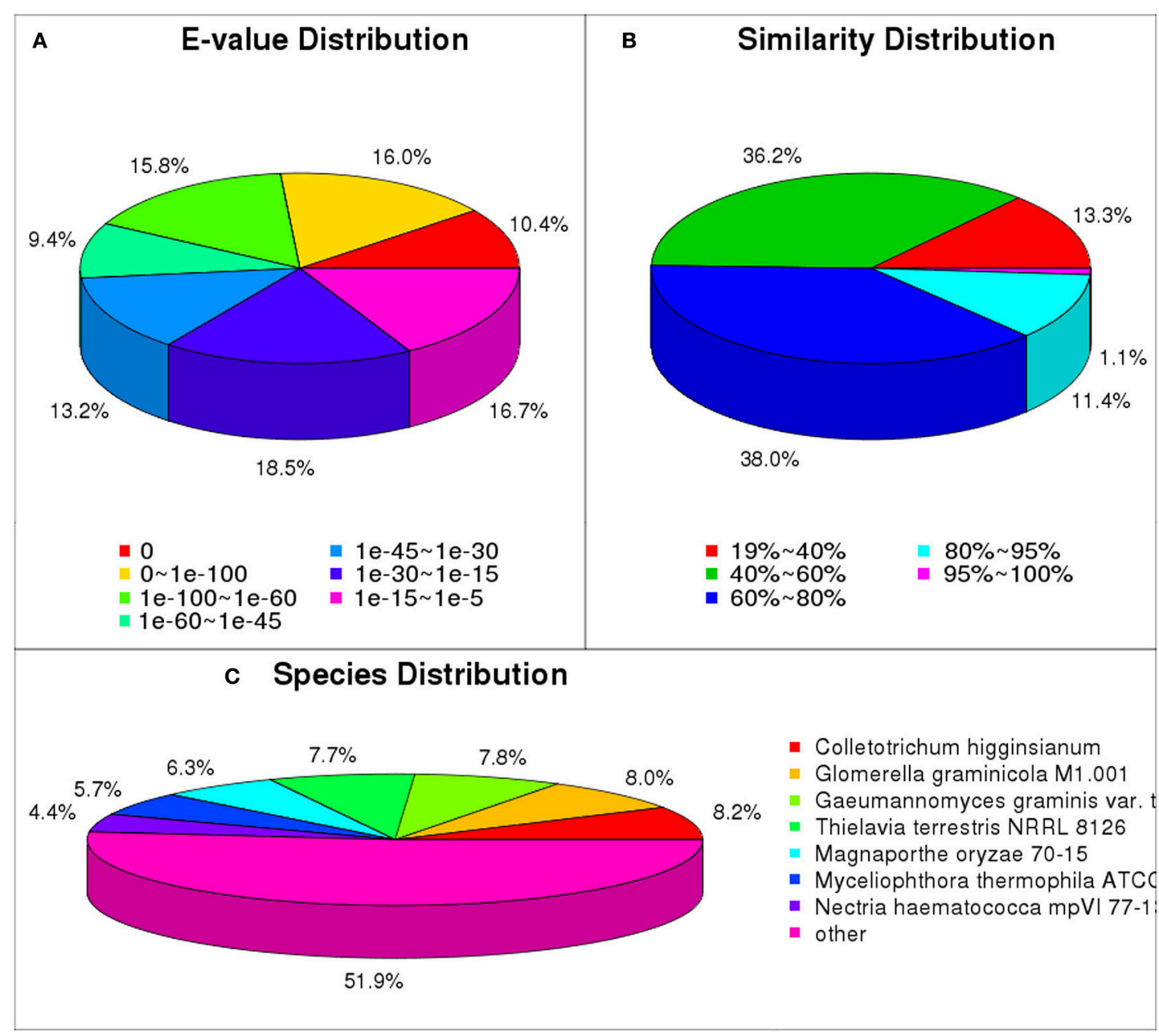

FIGURE 4 | NR classification of $\boldsymbol{P}$. liquidambari unigenes. (A) E-value distribution. (B) Identity distribution. (C) Species distribution. 

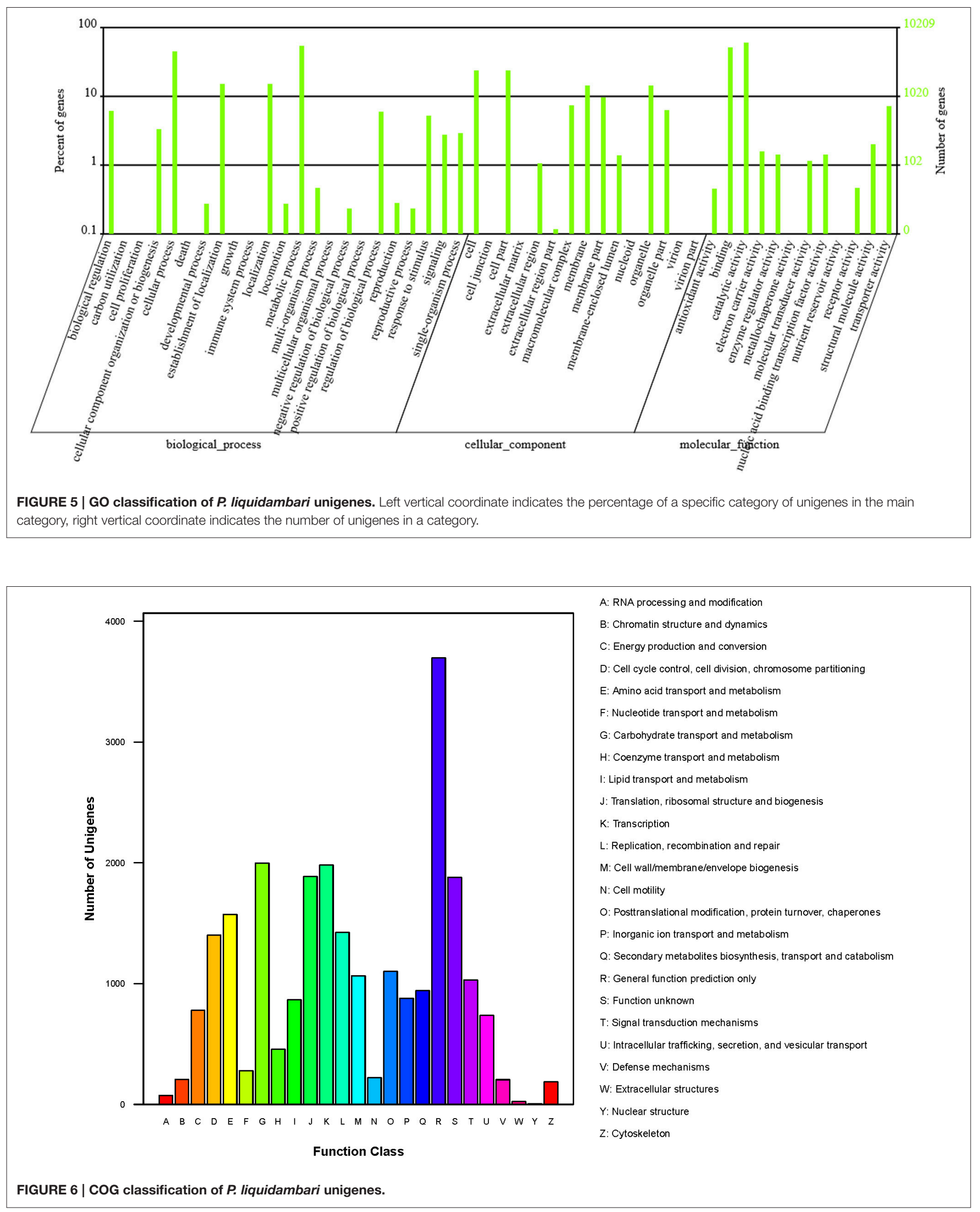
TABLE 2 | List of first 20 pathways with highest sequence numbers.

\begin{tabular}{|c|c|c|c|}
\hline Number & Pathway & $\begin{array}{c}\text { All genes with } \\
\text { pathway annotation } \\
(\mathbf{1 4 , 7 9 1 )}\end{array}$ & $\begin{array}{l}\text { Pathway } \\
\text { ID }\end{array}$ \\
\hline 1 & Metabolic pathways & 4785 (32.35\%) & ko01100 \\
\hline 2 & $\begin{array}{l}\text { Biosynthesis of secondary } \\
\text { metabolites }\end{array}$ & 2154 (14.56\%) & ko01110 \\
\hline 3 & Starch and sucrose metabolism & $1481(10.01 \%)$ & ko00500 \\
\hline 4 & RNA transport & $817(5.52 \%)$ & ko03013 \\
\hline 5 & $\begin{array}{l}\text { Amino sugar and nucleotide } \\
\text { sugar metabolism }\end{array}$ & $814(5.5 \%)$ & ko00520 \\
\hline 6 & Purine metabolism & $630(4.26 \%)$ & ko00230 \\
\hline 7 & MAPK signaling pathway - yeast & $596(4.03 \%)$ & ko04011 \\
\hline 8 & $\begin{array}{l}\text { Protein processing in } \\
\text { endoplasmic reticulum }\end{array}$ & $543(3.67 \%)$ & ko04141 \\
\hline 9 & Cell cycle - yeast & $467(3.16 \%)$ & ko04111 \\
\hline 10 & mRNA surveillance pathway & 445 (3.01\%) & ko03015 \\
\hline 11 & Meiosis - yeast & $414(2.8 \%)$ & ko04113 \\
\hline 12 & Spliceosome & 409 (2.77\%) & ko03040 \\
\hline 13 & Pyrimidine metabolism & $408(2.76 \%)$ & ko00240 \\
\hline 14 & RNA degradation & $370(2.5 \%)$ & ko03018 \\
\hline 15 & Lysine degradation & $365(2.47 \%)$ & ko00310 \\
\hline 16 & Tyrosine metabolism & $329(2.22 \%)$ & ko00350 \\
\hline 17 & Endocytosis & $281(1.9 \%)$ & ko04144 \\
\hline 18 & Glycolysis / Gluconeogenesis & $276(1.87 \%)$ & ko00010 \\
\hline 19 & $\begin{array}{l}\text { Ribosome biogenesis in } \\
\text { eukaryotes }\end{array}$ & $256(1.73 \%)$ & ko03008 \\
\hline 20 & $\begin{array}{l}\text { Fructose and mannose } \\
\text { metabolism }\end{array}$ & $249(1.68 \%)$ & ko00051 \\
\hline
\end{tabular}

three genes (putative alcohol dehydrogenase, cytochrome P450 monooxygenase and glycoside hydrolase family 72 protein) did not show consistent expression between qRT-PCR and RNAseq data sets. Comparison of data from Solexa sequencing analysis methods with data obtained from qRT-PCR indicates high credibility for these sequencing methods.

\section{DISCUSSION}

\section{Colonization of $P$. liquidambari in Rice}

To investigate the fate and behavior of $P$. liquidambari in rice in situ, B3 was tagged with the gfp gene. Colonization patterns of $P$. liquidambari were roughly divided into three successive time-space stages. First, extracellular colonization of runner hyphae outside the root, mainly concentrated in the base of the root hair, gradually formed a hyphal network on the root surface ( $<3$ dai) (Figures $\mathbf{1 A - F}$ and Video S1). Next, entering the biotrophic phase, intracellular and extracellular hyphae underwent branching growth along the root axis, and hyphae were extruded in a deformed fashion (4-10 dai) (Figures 1H-M), meaning that fungal infection began to be restricted. Finally, in the stage of colonization associated with programmed cell death, the vast majority of epidermal cells and part of the outer cortex cells were crowded with a large number of mycelium and sclerotium; the fungal structure

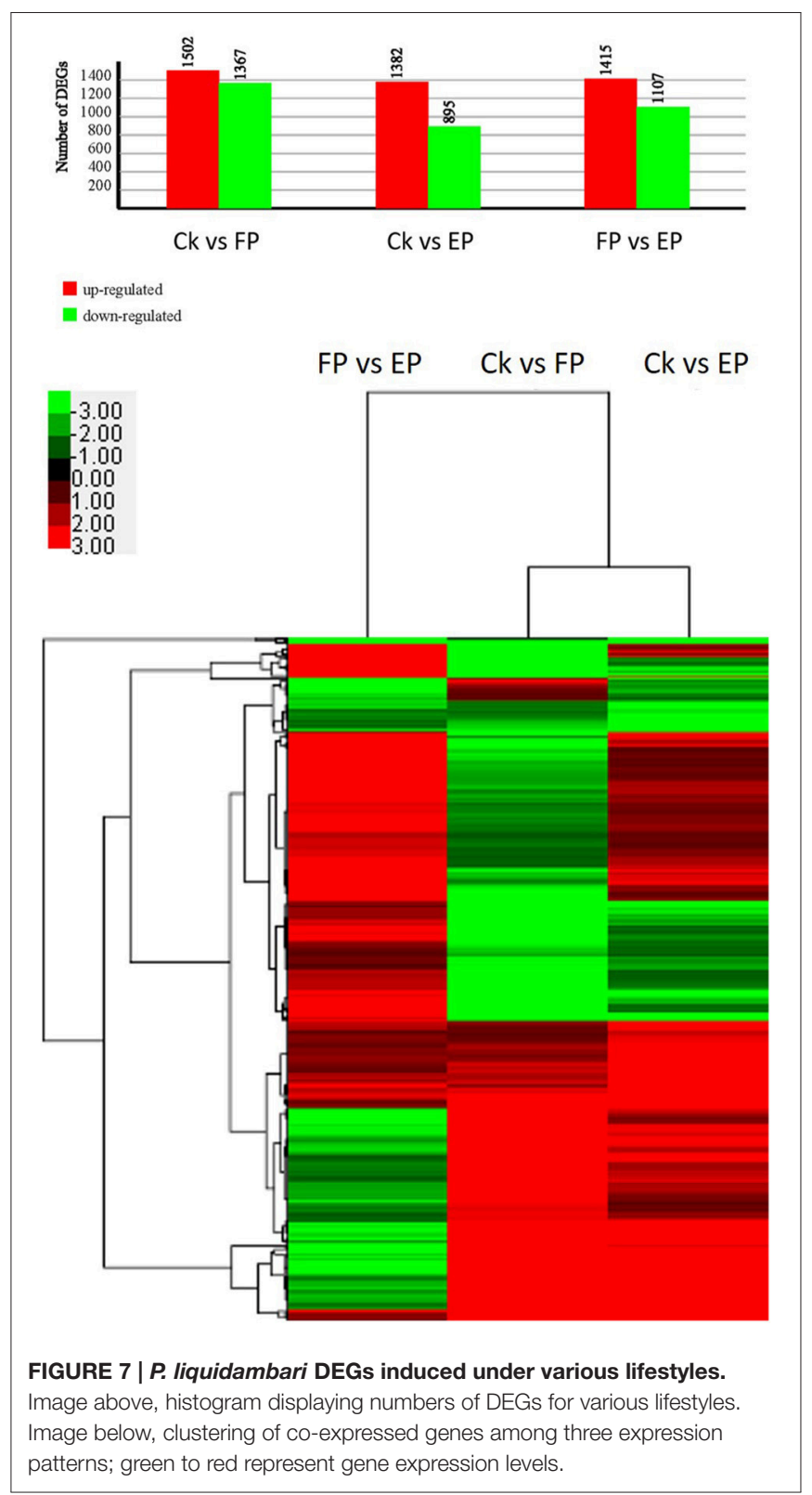

indicated that fungal infection was further blocked ( $>15$ dai) (Figures 1S,T). Interestingly, $P$. liquidambari still colonized when the host aged or died (Figures 1U-W). It is possible that $P$. liquidambari activated saprophytic programs to adapt to this variation. Colonization patterns of $P$. liquidambari in rice were different from Harpophora oryzae (Su et al., 2013), basidiomycete endophyte Piriformospora indica (Zuccaro et al., 2011; Lahrmann et al., 2013), soil invaders Fusarium equiseti, and Pochonia chlamydosporia (Maciá-Vicente et al., 2009), which belong to strict root endophytes. It was similar to Colletotrichum tofieldiae, in that a fraction of strong hypha penetrated and entered the root axis center, through the vascular bundle into the acrial part (Hiruma et al., 2016). During root infection, P. liquidambari generated a fungal structure similar to phytopathogen, with necklike constriction (Figure 1N), showing that any phytopathogen 


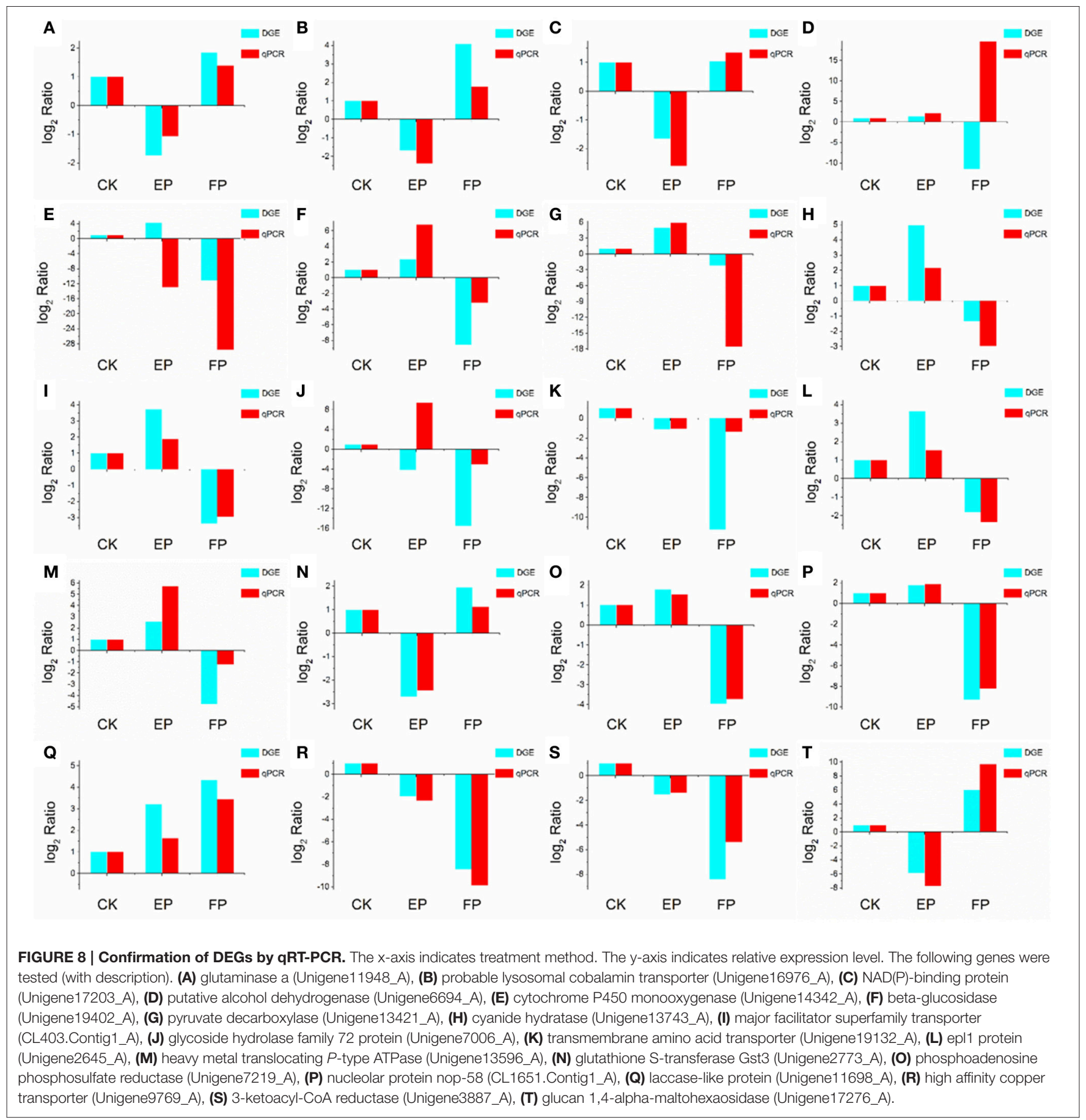

or endophyte can form similar infection structures during root infection. This phenomenon is related to infected tissue and thus belongs to tissue-specific infection. The most obvious similarity between $P$. liquidambari and phytopathogens is that they can infect vascular tissues and systematically spread to acrial parts through vascular tissue (Figures 1O-R).

In the colonization process of $P$. liquidambari, a large number of hyphae were limited to the epidermal layer and rhizosphere, and only a fraction of hyphae penetrated to the cortex. This fully demonstrates that $P$. liquidambari colonization is restricted by space and quantity. In late infection, $P$. liquidambari biomass remained at a steady state after hyphae entered the cortex cells, which can be explained by a reproduction rate of endophyte that was controlled within a certain range (Figure 2). In contrast, the reproduction of pathogen in the root was unrestricted and spread from roots to acrial parts, increasing biomass in an unrestricted fashion and inducing plant disease (Marcel et al., 2010; Su et al., 2013). In contrast, with this exploding 
pathogenic infection, the biomass of $P$. liquidambari in the host was maintained in an appropriate range without excessive reproduction. This is reminiscent of endophyte $H$. oryzae symbiosis with rice, initially showed moderate proliferation, subsequently colonization increased rapidly, finally reaching a steady-state level in rice roots (Su et al., 2013). Endophyte C. tofieldiae biomass was significantly increased in Trp-derived metabolites mutant, resulted in a severe negative effect on the growth of this mutant and eventually killed the plants (Hiruma et al., 2016). Likewise, the increased colonization of indolic glucosinolates mutant by root-associated fungi $P$. indica and Sebacina vermifera led in turn to plant death, suggesting compromised mutualism (Lahrmann et al., 2015). Therefore, another important difference between hostile interactions and mutualistic interactions is quantity rather than quality.

\section{Endophytic and Saprophytic Systems and Transcriptome Sequencing of $P$. liquidambari}

The transcriptome is the subset of genes active in tissues and species. To understand the dynamic of the transcriptome it is key to explain the phenotypic changes caused by combinations of genotype and environmental factors (Rockman and Kruglyak, 2006). Recently, Illumina RNA-seq has been used to identify genes of microbes related to plant interactions (Kawahara et al., 2012; O'connell et al., 2012; Alkan et al., 2015). RNA-seq can be used not only to detect organismic transcripts in existing genomic sequences but also to sequence non-model organisms lacking genomic information. To our knowledge, this is the first study of Phomopsis sp. using RNA-seq technology to obtain whole transcriptome information. Our experimental results provide more resources and sequences for studying filamentous endophyte $P$. liquidambari.

In the past, researchers have investigated the molecular genome of endophytes in vivo, but are challenged in retrieving endophyte gene information from high genetic background from the host plant. Compared to unrestrained pathogen reproduction after infection, endophytes steadily undergo symbiosis with the host and establish a subtle counterbalancing relationship that largely limits endophyte growth in the host. When host plants are used as vectors for transcriptome level research, a small number of expressed endophyte genes are typically disregarded due to the significant background of plant genes (PorrasAlfaro and Bayman, 2011). The callus is an undifferentiated living cell structure of plants that contains a set of defense systems similar to host plants (Nawrot-Chorabik et al., 2016). In this study, we used dual cultures of rice callus and $P$. liquidambari to simulate an endophytic environment in which organisms can release signals to recognize each other and form a relationship of simulated antagonism balance. Fungi growth on the callus surface can provide fungal hyphae directly, avoiding the interference from host cell. In litter culture, the humic acid substances of litter will significantly affect RNA quality. Thus, we adopted a litter liquid culture to suspend fungi in a liquid, collected the hyphae by flushing with sterile water and extracted RNA. The quality was improved using this method.
All transcriptome sequencing items were fit with the measurable indicators; Q20 percentage $>80 \%$, N percentage $<0.5 \%$ and GC percentage was $35-65 \%$, showing that the sequencing output and sequence quality were of good quality and could be further analyzed (Table 1).

\section{Divergent Expression Patterns from Amino Acid Metabolism to Fatty Acid Biosynthesis}

For our research, 108 biological pathways including the starch and sucrose metabolism pathway, amino sugar and nucleotide sugar metabolic pathways, the fatty acid biosynthesis pathway, and many others were identified by KEGG pathway analysis of unigenes. A total of 1638, 1330, and 1503 DEGs with pathway annotations were identified in the three respective contrast groups. From those pathways, we selected the fatty acid biosynthesis pathway, which is connected to amino acid metabolism and involved in carbohydrate metabolism, for deep analysis. The citrate cycle is the key pathway to energy metabolism. For P. liquidambari in the EP compared with FP, genes of the citrate cycle were differentially expressed, significantly enriched $(P<0.05)$, and nearly upregulated (Figure 9 and Table S7). Most genes for oxidative phosphorylation related to the citrate cycle were also upregulated. Carbon flux in the fatty acid biosynthesis pathway not only determines the component but also the content of fatty acids in fungi (Hao et al., 2014). Because alanine, aspartate and glutamate metabolism are closely connected to the citrate cycle, and thus genes for these pathways are also enriched and mostly up-regulated. The genes of glycine, serine and threonine metabolism and cysteine and methionine metabolism were up-regulated. The common product of these amino acids metabolisms is pyruvate, and a supply of acetyl-CoA plays a more important role in fungal fatty acid biosynthesis. In Mortierella alpina, tyrosine and phenylalanine were considered to contribute NADPH and acetyl-CoA to lipid metabolism through a phenylalanine-hydroxylating system (Wang et al., 2013).

Fatty acid synthesis and transformation plays an important role in fungal growth and development. During intraradical growth, much fatty acid synthesis is required for lipid storage and membrane proliferation of fungi. Adaptation of lipid metabolism may be the prerequisite for symbiosis to achieve function compatibility between fungi and periarbuscular membrane. Fungi were forced to change their membrane lipid composition to allow nutrient exchange between fungal arbuscular and plant periarbuscular membranes (Wewer et al., 2014). In the EP, compared to FP, nine DEGs associated with fatty acid biosynthesis were co-expressed. Interestingly, two identified unigenes were identical to those identified from the GO analysis. Among those nine DEGs, only one unigene (Unigene2899_A) was down-regulated; this unigene encodes 3-oxoacyl-[acylcarrier protein] reductases (FabG). The other eight unigenes were up-regulated and may be positively regulated genes in the fatty acid biosynthesis pathway. Figure 9 shows the locations of DEGs in the fatty acid biosynthesis pathway. The many up-regulated genes indicate that more positively controlled 


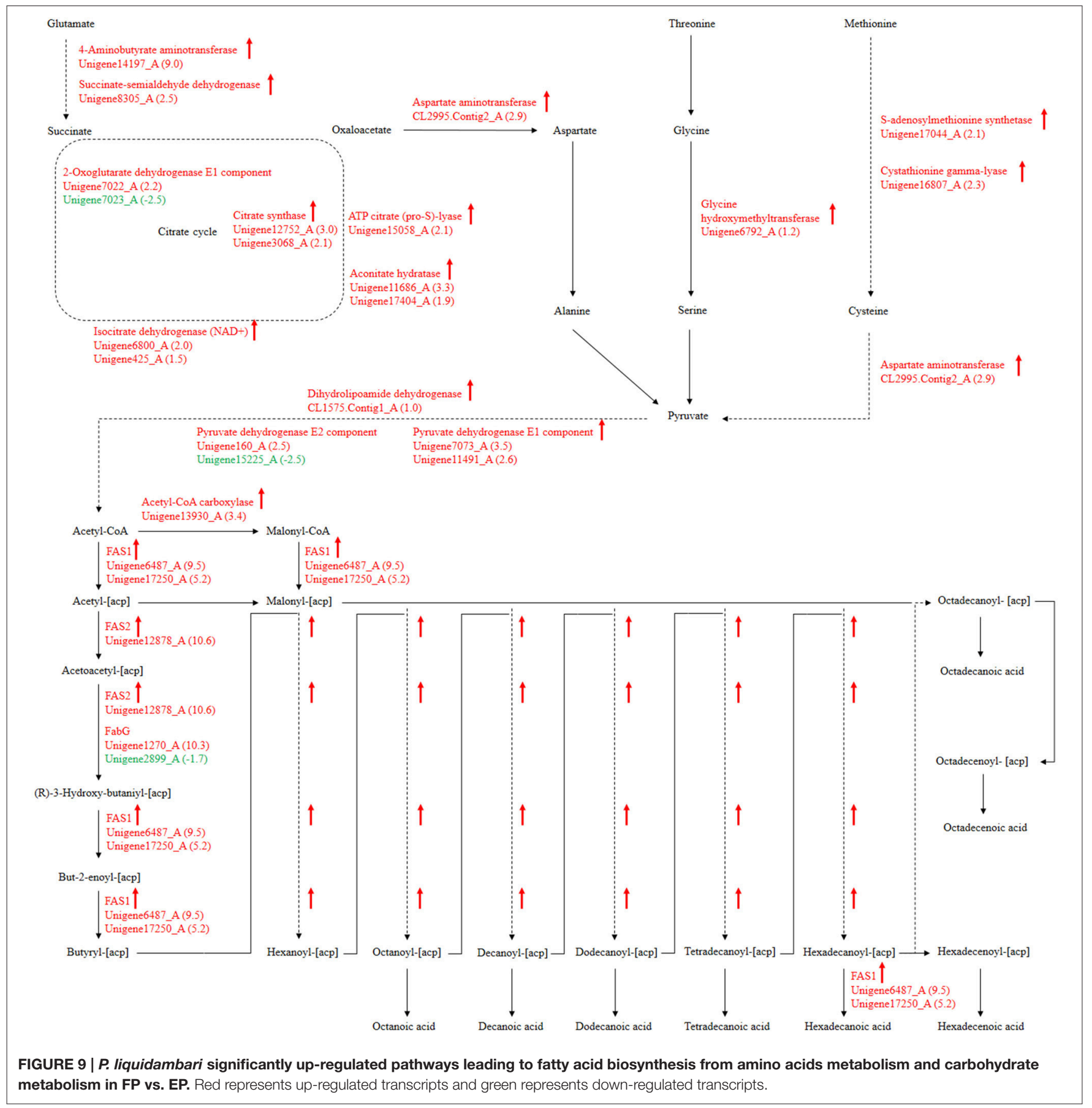

genes than negatively regulated genes function in fatty acid biosynthesis.

Polyunsaturated fatty acids or oxylipins can trigger extensive cellular responses, such as pathogenicity arsenals, defense and stress response, secondary metabolism, oxylipin synthesis and cell wall formation. This indicates that generation and recognition are important for coordinating these responses, which can guide pathogen adaptation to host response (Tsitsigiannis and Keller, 2007). Fungal oxylipin repertoire may participate in the competition between pathogen and host and is also involved in reproduction and development (Oliw et al., 2016). Recent evidence has showed that fatty acids also appear and play a role in beneficial plant-fungi interactions. The endophyte Fusarium incarnatum in the embryo of Aegiceras corniculatum can produce archetypal plant defense oxylipins that can protect the embryo and are derived from linoleic acid (Pohl and Kock, 2014). Esterified fatty acids of Lasiodiplodia theobromae can be used as plant growth regulators in tobacco 
and have similar activity to gibberellic acid (Uranga et al., 2016). In addition, commensal Candida albicans produce a low-level of resolvin E1, an eicosanoids that works as an effective antiinflammatory lipid and can inhibit adaptive immune responses and protect commensal yeast from host immune attacks (Pohl and Kock, 2014). DGE data show that genes encoding the FAS1, FAS2, and Acetyl-CoA carboxylase of fatty acid synthesis were up-regulated in EP. FabG genes were both up- and downregulated (Figure 9). There are considerable differences in fatty acid synthesis between the symbiotic and asymbiotic states. Lipid compounds play a key role in symbiotic signals and are likely involved in signaling communication between plants and endophytes. Oxidized fatty acids as signaling molecules have an ancient evolutionary origin and are ideal candidates for inter-kingdom signaling communication (Pohl and Kock, 2014). Oxylipins as intracellular and intercellular communication signals showed vital bioactivities in fungi, plants and animals. The oxylipin signature profile of fungi serves an adjusting function as a "master switch" under different environmental conditions and provides the appropriate mechanisms to microbes by balancing meiospore and mitospore development temporarily. On the basis of Aspergillus-seed pathosystems, as supported by data, oxylipin cross-talk is reciprocal. The structural similarity of plant and fungal oxylipins has given rise to a hypothesis that they are important molecules in cross-kingdom communication (Tsitsigiannis and Keller, 2007).

\section{Differences in Secondary Metabolism from Terpenoid to Steroid Biosynthesis}

Secondary metabolism is strictly regulated by fungi and is often closely related to asexual reproduction. Secondary metabolites produced from Trichoderma include pyrone, antimicrobial peptides and terpenoids that can inhibit the growth of phytopathogens. Several studies have reported that endophytes are involved in the synthesis of plant secondary metabolites during symbiosis with plants. For example, as Phoma Medicaginis switches from the endophytic stage to the saprophytic stage, a large increase in the production of brefeldin A contributes to host defense competitive saprophytes. Low levels of these compounds will inhibit the defense system to maintain the endophytic state of P. Medicaginis (Weber et al., 2004). Endophytes can affect growth processes by influencing secondary metabolism under different habitats. Genes encoding secondary metabolism in Epichloe spp., C. tofieldiae and $H$. oryzae were significantly expressed, but strongly reduced in sebacinales, indicating convergent adaptation to a life inside living host cells (Zuccaro et al., 2011; Fesel and Zuccaro, 2016). Cytochrome P450 monooxygenases play diverse and vital roles in various metabolisms and when fungi adapt to specific ecological niches (Chen et al., 2014). As shown in Table 3, compared with FP, the corresponding genes of secondary metabolites synthesis pathways (e.g., stilbenoid, diarylheptanoid and gingerol biosynthesis, phenylpropanoid biosynthesis, terpenoid backbone biosynthesis, ubiquinone and other terpenoid-quinone biosynthesis), were up-regulated. Most of these genes coded for the cytochrome P450 enzyme family and revealed that various cytochrome P450s are involved when filamentous fungi generate a large number of secondary metabolites. Similarly, a phylogenetic analysis revealed the specific expansion of secondary metabolite synthesis genes in $H$. oryzae, as well as cytochrome $\mathrm{P} 450$ monooxygenases (Xu et al., 2014). Cytochrome P450 catalyzes biosynthetic metabolism of endogenous substances with important physiological functions such as fatty acids, terpenoids and hormones, and thus $\mathrm{P} 450$ plays an important role in the modification of secondary metabolites. In tryptophan metabolism, tryptophan is converted into indole derivatives through these cytochrome P450 enzymes and further forms various secondary metabolites. Several secondary metabolisms originating from tryptophan were essential for beneficial symbiosis with C. tofieldiae. Mutation of the organism not only ended this beneficial symbiotic relationship, but

TABLE 3 | Upregulation of genes involved in biosynthesis of secondary metabolites in FP vs. EP.

\begin{tabular}{lllc}
\hline Category or unigene ID & Gene ID & Annotation & Accession no. \\
\hline Unigene14342_A & 145246720 & cytochrome P450 monooxygenase & XP_001395609.1 \\
Unigene113_A & 310795890 & cytochrome P450 & EFQ31351.1 \\
Unigene13908_A & 302415555 & cytochrome P450 monooxygenase & XP_003005609.1 \\
Unigene6720_A & 310789565 & oxidoreductase NAD-binding domain-containing protein & EFQ25098.1 \\
Unigene10987_A & 322705062 & branched-chain-amino-acid aminotransferase & 14.26 \\
Unigene3485_A & 16416002 & probable cytochrome P450 monooxygenase (lovA) & EFY96651.1 \\
Unigene19402_A & 302423734 & beta-glucosidase & CAB91316.2 \\
Unigene2980_A & 347839081 & similar to cytochrome P450 monooxygenase & XP_003009697.1 \\
Unigene57_A & 52548220 & C-5 sterol desaturase C-like & CCD53653.1 \\
Unigene14360_A & 400600304 & Cytochrome P450 CYP505D4 & AAU82099.1 \\
Unigene10045_A & 380493536 & trichothecene C-8 hydroxylase & 12.04 \\
Unigene1270_A & 358369448 & 3-ketoacyl-acyl carrier protein reductase & 10.93 \\
Unigene19132_A & 380492609 & transmembrane amino acid transporter & 10.88 \\
Unigene7296_A & 240282093 & Cytochrome P450 & 10.77 \\
Unigene17476_A & 380488167 & CTP synthase & CCF33809.1 \\
Unigene19143_A & 380479107 & benzoate 4-monooxygenase cytochrome P450, partial & GAA86062.1 \\
& & CCF34481.1 & 10.50 \\
\end{tabular}


increased colonization of $C$. tofieldiae such that it eventually killed the host plant (Hiruma et al., 2016). DGE showed that the corresponding genes in the pathway of sesquiterpenoid and triterpenoid biosynthesis were significantly up-regulated $(P<$ 0.05) (Figure 10 and Table S7). This is consistent with a previous study that shoed that endophyte Gilmaniella sp. AL12 can establish symbiosis with Atractylodes lancea and greatly promotes terpenoids accumulation in the herb (Yuan et al., 2016). We found two up-regulated genes in EP: farnesyl-diphosphate farnesyl transferase (Unigene14286_A), involved in isoprenoid biosynthesis, and squalene monooxygenase (Unigene13115_A). Both enzymes have oxidoreductase activity and effect secondary metabolites synthesis and plant-endophyte symbiosis. They also participated in steroid biosynthesis in lipid metabolism. Cytochrome P450 also plays housekeeping functions in fungi. For instance, CYP51 takes part in sterol biosynthesis and is a popular antifungal target to control fungal disease in humans and crops (Becher and Wirsel, 2012). Previous studies have shown that CYP51 and CYP61 play housekeeping functions in the sterol biosynthesis of filamentous fungi (Kelly et al., 2009). The personalized cytochrome P450 components of fungi indicate that highly specialized functions enable evolutionary adaptation to ecological niches.

\section{Distinct Xenobiotic Biodegradation and Metabolism}

Our previous studies have reported the capacity of $P$. liquidambari to decompose phenolic acids, cellulose, $\mathrm{N}$-heterocyclic indole and the polycyclic aromatic hydrocarbon phenanthrene (Dai et al., 2010a,b; Chen et al., 2011, 2013b,c). The cytochrome P450 family also contributes to ecological functions as a decomposer or saprophyte. For example, the cytochrome P450s of white-rot fungi Phanerochaete chrysosporium is involved in vast xenobiotic biodegradation of extensive environmental toxic chemicals and the natural aromatic polymer lignin (Syed and Yadav, 2012). The diversity of cytochrome P450s may be closely related to fungal survival environment. For example, the cytochrome P450s of white-rot and brown-rot fungi break down plant materials in the environment (Eastwood and Watkinson, 2011; Chen et al., 2014). As DGE showed that cytochrome P450s are involved in xenobiotic biodegradation, we speculated that endophyte $P$. liquidambari appropriately biodegraded harmful xenobiotics or used them as carbon sources to adapted to the host after entering. In addition, endophytes promote production of secondary metabolites that are beneficial to the host and enable both the endophytes and host plants both to grow.

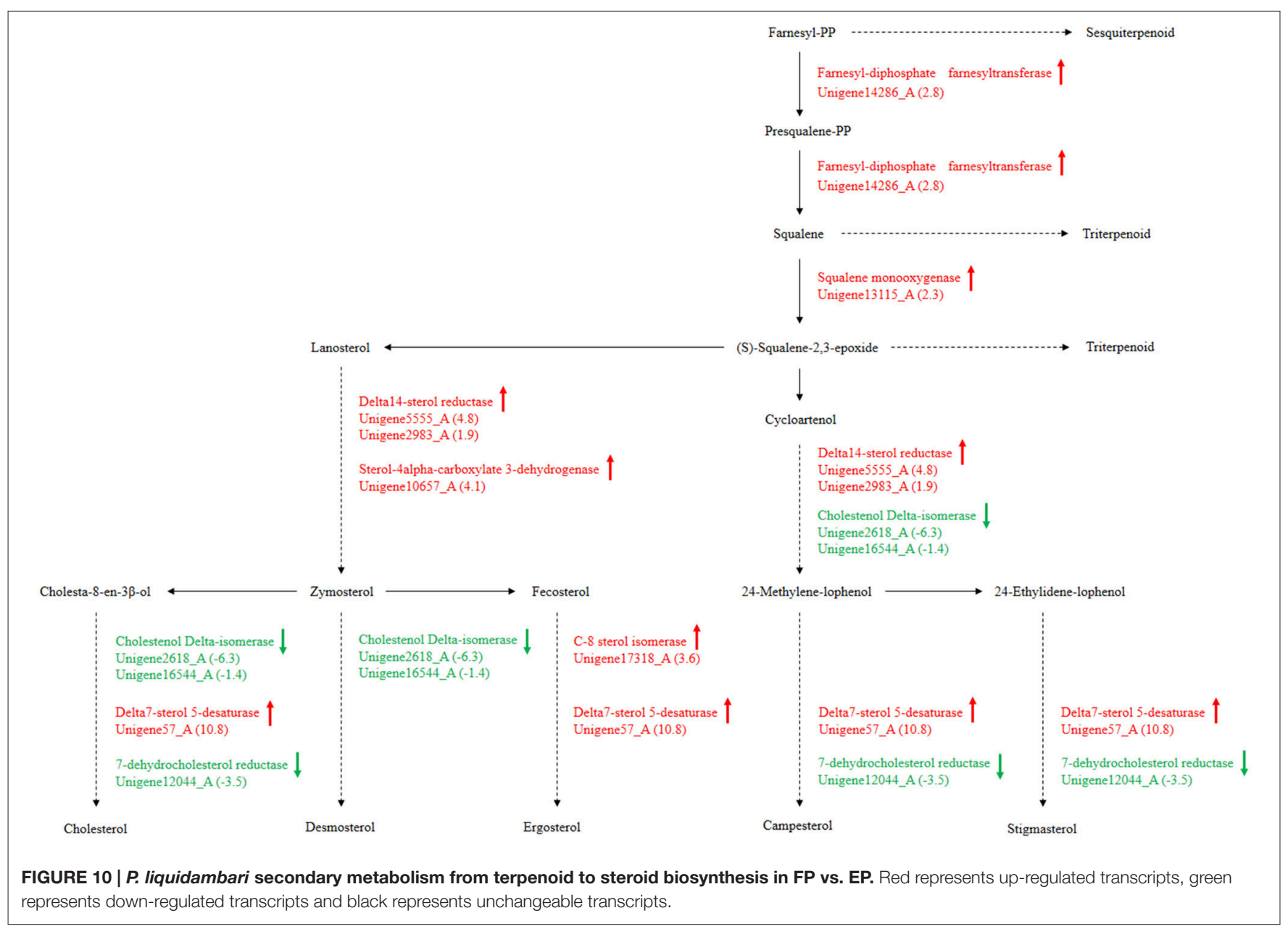


TABLE 4 | Expression profiles of pathways involved in xenobiotics biodegradation in FP vs. EP.

\begin{tabular}{|c|c|c|c|c|c|}
\hline $\begin{array}{l}\text { Enzyme } \\
\text { name }\end{array}$ & Orthology & $\begin{array}{c}\text { Numbers } \\
\text { of } \\
\text { unigene }\end{array}$ & $\begin{array}{l}\text { Numbers of } \\
\text { up-regulated } \\
\text { gene }\end{array}$ & $\begin{array}{c}\text { Numbers of } \\
\text { down-regulated } \\
\text { gene }\end{array}$ & Expression \\
\hline \multicolumn{6}{|c|}{ BISPHENOL DEGRADATION } \\
\hline 1.14.-.- & K00517 & 23 & 14 & 9 & Up \\
\hline 4.2.1.- & K01726 & 1 & 0 & 1 & Down \\
\hline 1.14.13.- & K00492 & 4 & 2 & 2 & \\
\hline 1.13.-.- & K05915 & 1 & 0 & 1 & Down \\
\hline 1.1.1.- & K00100 & 9 & 1 & 8 & Down \\
\hline 3.1.1.- & K01066 & 2 & 1 & 1 & \\
\hline \multicolumn{6}{|c|}{ CHLOROALKANE AND CHLOROALKENE DEGRADATION } \\
\hline 1.1.1.1 & $\begin{array}{l}\text { K13953; } \\
\text { K00121 }\end{array}$ & 6 & 1 & 5 & Down \\
\hline 1.2.1.3 & K00128 & 1 & 0 & 1 & Down \\
\hline 3.8.1.- & K01564 & 2 & 1 & 1 & \\
\hline 1.1.1.- & K00100 & 9 & 1 & 8 & Down \\
\hline 3.8.1.2 & K01560 & 1 & 1 & 0 & Up \\
\hline \multicolumn{6}{|c|}{ CAPROLACTAM DEGRADATION } \\
\hline 1.14 .13 .22 & K03379 & 1 & 0 & 1 & Down \\
\hline 3.1 .1 .17 & K01053 & 1 & 0 & 1 & Down \\
\hline 1.1.1.2 & K00002 & 2 & 0 & 2 & Down \\
\hline \multicolumn{6}{|c|}{ POLYCYCLIC AROMATIC HYDROCARBON DEGRADATION } \\
\hline 1.3.1.- & K00224 & 3 & 0 & 3 & Down \\
\hline $1.14 .-\cdot-$ & K00517 & 23 & 14 & 9 & Up \\
\hline 1.14.13.1 & K00480 & 4 & 1 & 3 & Down \\
\hline 1.14.13.- & K00492 & 4 & 2 & 2 & \\
\hline 2.1.1.- & K00599 & 2 & 1 & 1 & \\
\hline \multicolumn{6}{|c|}{ NAPHTHALENE DEGRADATION } \\
\hline 1.14.13.- & K00492 & 4 & 2 & 2 & \\
\hline 1.14.13.1 & K00480 & 4 & 1 & 3 & Down \\
\hline 1.13.--- & K05915 & 1 & 0 & 1 & Down \\
\hline 4.2.1.- & K01726 & 1 & 0 & 1 & Down \\
\hline 1.2.1.- & K00155 & 1 & 0 & 1 & Down \\
\hline 1.1 .1 .1 & $\begin{array}{l}\text { K13953; } \\
\text { K00121 }\end{array}$ & 6 & 1 & 5 & Down \\
\hline \multicolumn{6}{|c|}{ CHLOROCYCLOHEXANE AND CHLOROBENZENE DEGRADATION } \\
\hline 3.1 .1 .45 & K01061 & 3 & 1 & 2 & \\
\hline 1.14.13.- & K00492 & 4 & 2 & 2 & \\
\hline 3.8.1.2 & K01560 & 1 & 1 & 0 & Up \\
\hline 1.14.13.7 & K03380 & 1 & 0 & 1 & Down \\
\hline 1.3.1.- & K00224 & 3 & 0 & 3 & Down \\
\hline \multicolumn{6}{|c|}{ AMINOBENZOATE DEGRADATION } \\
\hline 3.5 .1 .4 & K01426 & 1 & 0 & 1 & Down \\
\hline 1.14 .13 .12 & K07824 & 3 & 3 & 0 & Up \\
\hline 1.14.-.- & K00517 & 23 & 14 & 9 & Up \\
\hline 1.14.13.7 & K03380 & 1 & 0 & 1 & Down \\
\hline 3.1.1.- & K01066 & 2 & 1 & 1 & \\
\hline 3.1.3.1 & $\begin{array}{l}\text { K01113; } \\
\text { K01077 }\end{array}$ & 3 & 0 & 3 & Down \\
\hline 3.1.3.2 & K01078 & 1 & 0 & 1 & Down \\
\hline 1.14.13.- & K00492 & 4 & 2 & 2 & \\
\hline 2.3.1.- & K00680 & 5 & 4 & 1 & Up \\
\hline
\end{tabular}

(Continued)
TABLE 4 | Continued

\begin{tabular}{|c|c|c|c|c|c|}
\hline $\begin{array}{l}\text { Enzyme } \\
\text { name }\end{array}$ & Orthology & $\begin{array}{c}\text { Numbers } \\
\text { of } \\
\text { unigene }\end{array}$ & $\begin{array}{l}\text { Numbers of } \\
\text { up-regulated } \\
\text { gene }\end{array}$ & $\begin{array}{c}\text { Numbers of } \\
\text { down-regulated } \\
\text { gene }\end{array}$ & Expression \\
\hline 1.14.14.1 & $\begin{array}{l}\text { K14338; } \\
\text { K00493 }\end{array}$ & 2 & 1 & 1 & \\
\hline \multicolumn{6}{|c|}{ STYRENE DEGRADATION } \\
\hline 3.5.1.4 & K01426 & 1 & 0 & 1 & Down \\
\hline 1.13 .11 .5 & K00451 & 1 & 0 & 1 & Down \\
\hline 3.7.1.2 & K01555 & 1 & 0 & 1 & Down \\
\hline \multicolumn{6}{|c|}{ FLUOROBENZOATE DEGRADATION } \\
\hline 3.1 .1 .45 & K01061 & 3 & 1 & 2 & \\
\hline \multicolumn{6}{|c|}{ ATRAZINE DEGRADATION } \\
\hline 3.8.1.- & K01564 & 2 & 1 & 1 & \\
\hline \multicolumn{6}{|c|}{ DIOXIN DEGRADATION } \\
\hline 1.14.13.1 & K00480 & 4 & 1 & 3 & Down \\
\hline \multicolumn{6}{|c|}{ TOLUENE DEGRADATION } \\
\hline 1.14.13.7 & K03380 & 1 & 0 & 1 & Down \\
\hline 1.14.13.- & K00492 & 4 & 2 & 2 & \\
\hline 3.1 .1 .45 & K01061 & 3 & 1 & 2 & \\
\hline \multicolumn{6}{|c|}{ BENZOATE DEGRADATION } \\
\hline 1.14 .13 .12 & K07824 & 3 & 3 & 0 & Up \\
\hline 4.2.1.- & K01726 & 1 & 0 & 1 & Down \\
\hline 2.3.1.16 & K00632 & 1 & 1 & 0 & Up \\
\hline 2.3.1.- & K00680 & 5 & 4 & 1 & Up \\
\hline 2.3.1.9 & K00626 & 1 & 0 & 1 & Down \\
\hline \multicolumn{6}{|c|}{ ETHYLBENZENE DEGRADATION } \\
\hline 2.3.1.16 & K00632 & 1 & 1 & 0 & Up \\
\hline 2.3.1.- & K00680 & 5 & 4 & 1 & Up \\
\hline \multicolumn{6}{|c|}{ METABOLISM OF XENOBIOTICS BY CYTOCHROME P450 } \\
\hline 3.3.2.9 & K01253 & 1 & 0 & 1 & Down \\
\hline 2.5.1.18 & K00799 & 1 & 1 & 0 & Up \\
\hline 1.1.1.1 & $\begin{array}{l}\text { K13953; } \\
\text { K00121 }\end{array}$ & 6 & 1 & 5 & Down \\
\hline 1.2 .1 .5 & K00129 & 1 & 1 & 0 & Up \\
\hline \multicolumn{6}{|c|}{ DRUG METABOLISM - CYTOCHROME P450 } \\
\hline 1.14.13.8 & K00485 & 5 & 1 & 4 & Down \\
\hline 2.5.1.18 & K00799 & 1 & 1 & 0 & Up \\
\hline 1.1.1.1 & $\begin{array}{l}\text { K13953; } \\
\text { K00121 }\end{array}$ & 6 & 1 & 5 & Down \\
\hline 1.2 .1 .5 & K00129 & 1 & 1 & 0 & Up \\
\hline
\end{tabular}

However, endophytes affected by defensive responses and host plant metabolites cannot overgrow at large scales, resulting in a subtle symbiotic relationship.

It has been increasingly demonstrated that endophytes that quickly decompose plant litter in vitro can initiate saprophytic effects when the endophytic survival environment is destroyed, for example through plant senescence or falling. This saprophytic effect is similar to a saprophytic lifestyle that maintains survival and growth by metabolizing compounds in litter that are usually difficult to decompose. In this study, we found that the expression of partial genes involved in xenobiotic biodegradation and metabolism of $P$. liquidambari in FP was up-regulated by 
DGE. As shown in Table 4, in EP compared with FP, genes of pathways concerning bisphenol degradation, chloroalkane and chloroalkene degradation, caprolactam degradation, polycyclic aromatic hydrocarbon degradation, naphthalene degradation, chlorocyclohexane and chlorobenzene degradation, aminobenzoate degradation, styrene degradation, fluorobenzoate degradation, atrazine degradation, dioxin degradation, toluene degradation, benzoate degradation, ethylbenzene degradation, metabolism of xenobiotics by cytochrome P450 and drug metabolism by cytochrome P450 were both up- and downregulated. This indicates that $P$. liquidambari can decompose heterocyclic compounds in both endophytic and saprophytic lifestyles. However, most genes are down-regulated in FP vs. $\mathrm{EP}$, indicating that the ability of $P$. liquidambari to degrade aromatic or phenolic compounds was enhanced in a saprophytic lifestyle. Endophyte P. liquidambari in a simulated saprophytic environment that lacked nutrition that can be utilized directly forced the fungus to decompose residual organic matter in the litter. Lignin, a main component of litter, was also utilized by the fungus because the corresponding genes for biodegradation of xenobiotics such as bisphenol via lignin degradation were up-regulated. This further verified previous research by Dai et al. (2010b), who found that endophytes can decompose polycyclic aromatic hydrocarbons in vitro. Chen et al. (2013a) reported that application of endophyte $P$. liquidambari to soil observably promoted the release of inorganic nitrogen through organic matter degradation. Co-culture of P. liquidambari with indole and litter increased indole degradation significantly: $99.1 \%$ of indole was removed after $60 \mathrm{~h}$ of cultivation, and residual indole levels were below the detection threshold at the $84 \mathrm{~h}$ time point (Chen et al., 2013c). Zhou et al. (2014a,b) utilized food waste and wheat straw as nutrient sources in a simulated saprophytic system of cultured P. liquidambari. The fermentation product was applied to continuously cropped peanut soil, and the concentrations of vanillic acid, coumaric acid, and 4-hydroxybenzoic acid in soil had decreased by 52.5 , 49.4 , and $57.4 \%$, respectively, after 28 days. The bacterial and fungal community structures in the rhizosphere soil were affected by changes in phenolic acid concentration and promoted peanut seedling growth and nodulation. These changes demonstrate the application prospects for P. liquidambari in the decomposition of difficult-to-decompose organic compounds and environmental remediation. In addition, the results indicate the advantages of nutrient restoration to successive cropping of farmland, when plant residue exempt from plowing can thoroughly decompose.

\section{AUTHOR CONTRIBUTIONS}

JZ performed most of the work, including experimental design and operation, data analysis and manuscript writing. XL and YC prepared samples and extracted fungal RNA. CD supervised all work.

\section{ACKNOWLEDGMENTS}

We are grateful to the National Natural Science Foundation of China (NSFC NO. 31570491), a project funded by the Priority Academic Program Development of the Jiangsu Higher Education Institutions, the Research Fund of the State Key Laboratory of Soil and Sustainable Agriculture, Nanjing Institute of Soil Science, Chinese Academy of Science (Y412201435) and the Graduate Research and Innovation Project of Jiangsu Province (KYLX16_1282) for their financial support.

\section{SUPPLEMENTARY MATERIAL}

The Supplementary Material for this article can be found online at: http://journal.frontiersin.org/article/10.3389/fpls.2017. 00121/full\#supplementary-material

Table S1 | qPCR primer sequences.

Table S2 | Length distribution of contigs.

Table S3 | Length distribution of unigenes.

Table S4 | Functional annotation of unigenes.

Table S5 | GO classification of P. liquidambari unigenes.

Table S6 | KEGG annotation of $P$. liquidambari transcriptome.

Table S7 | Significantly enriched pathway in Ck vs. EP, Ck vs. FP, and FP vs. EP.

Figure S1 | Rice callus.

Figure S2 | Length distribution of CDS for unigenes predicted by BLAST and ESTScan. Length distribution of nucleotide sequence (A) and protein sequence (B) predicted by BLAST and length distribution of nucleotide sequence (C) and protein sequence (D) predicted by ESTScan. Horizontal coordinates are sequence size and vertical coordinates are numbers of unigenes.

Video S1 | Three-dimensional scanning for cross-section of root tip, runnner hypha interweaved to form hyphal network.

\section{REFERENCES}

Alkan, N., Friedlander, G., Ment, D., Prusky, D., and Fluhr, R. (2015). Simultaneous transcriptome analysis of Colletotrichum gloeosporioides and tomato fruit pathosystem reveals novel fungal pathogenicity and fruit defense strategies. New Phytol. 205, 801-815. doi: 10.1111/nph.13087

Audic, S., and Claverie, J. M. (1997). The significance of digital gene expression profiles. Genome Res. 7, 986-995. doi: 10.1101/gr.7.10.986

Becher, R., and Wirsel, S. G. R. (2012). Fungal cytochrome P450 sterol $14 \alpha-$ demethylase (CYP51) and azole resistance in plant and human pathogens. Appl. Microbiol. Biotechnol. 95, 825-840. doi: 10.1007/s00253-012-4195-9

Chen, W., Lee, M. K., Jefcoate, C., Kim, S. C., Chen, F., and Yu, J. H. (2014). Fungal cytochrome p450 monooxygenases: their distribution, structure, functions, family expansion, and evolutionary origin. Genome Biol. Evol. 6, 1620-1634. doi: 10.1093/gbe/evu132

Chen, Y., Peng, Y., Dai, C.-C., and Ju, Q. (2011). Biodegradation of 4 hydroxybenzoic acid by Phomopsis liquidambari. Appl. Soil Ecol. 51, 102-110. doi: 10.1016/j.apsoil.2011.09.004

Chen, Y., Ren, C. G., Yang, B., Peng, Y., and Dai, C. C. (2013a). Priming effects of the endophytic fungus Phomopsis liquidambari on soil mineral N transformations. Microb. Ecol. 65, 161-170. doi: 10.1007/s00248-0120093-z 
Chen, Y., Wang, H. W., Li, L., and Dai, C. C. (2013b). The potential application of the endophyte Phomopsis liquidambari to the ecological remediation of longterm cropping soil. Appl. Soil Ecol. 67, 20-26. doi: 10.1016/j.apsoil.2013.02. 004

Chen, Y., Xie, X. G., Ren, C. G., and Dai, C. C. (2013c). Degradation of Nheterocyclic indole by a novel endophytic fungus Phomopsis liquidambari. Bioresour. Technol. 129, 568-574. doi: 10.1016/j.biortech.2012.11.100

Conesa, A., Götz, S., García-Gómez, J. M., Terol, J., Talón, M., and Robles, M. (2005). Blast2GO: a universal tool for annotation, visualization and analysis in functional genomics research. Bioinformatics 21, 3674-3676. doi: 10.1093/bioinformatics/bti610

Dai, C. C., Chen, Y., Tian, L. S., and Shi, Y. (2010a). Correlation between invasion by endophytic fungus Phomopsis sp and enzyme production. Afr. J. Agric. Res. 5, 1324-1330. doi: 10.5897/AJAR09.541

Dai, C. C., Tian, L. S., Zhao, Y. T., Chen, Y., and Xie, H. (2010b). Degradation of phenanthrene by the endophytic fungus Ceratobasidum stevensii found in Bischofia polycarpa. Biodegradation 21, 245-255. doi: 10.1007/s10532-0099297-4

Eastwood, D. C., and Watkinson, S. C. (2011). The plant cell wall-decomposing machinery underlies the functional diversity of forest fungi. Science 333, 762-765. doi: 10.1126/science.1205411

Fesel, P. H., and Zuccaro, A. (2016). Dissecting endophytic lifestyle along the parasitism/mutualism continuum in Arabidopsis. Curr. Opin. Microbiol. 32, 103-112. doi: 10.1016/j.mib.2016.05.008

Grabherr, M. G., Haas, B. J., Yassour, M., Levin, J. Z., Thompson, D. A., Amit, I., et al. (2011). Full-length transcriptome assembly from RNA-Seq data without a reference genome. Nat. Biotechnol. 29, 644-652. doi: 10.1038/ nbt.1883

Hao, G., Chen, H., Wang, L., Gu, Z., Song, Y., Zhang, H., et al. (2014). Role of malic enzyme during fatty acid synthesis in the oleaginous fungus Mortierella alpina. Appl. Environ. Microbiol. 80, 2672-2678. doi: 10.1128/AEM.00140-14

Hiruma, K., Gerlach, N., Sacristan, S., Nakano, R. T., Hacquard, S., Kracher, B., et al. (2016). Root endophyte colletotrichum tofieldiae confers plant fitness benefits that are phosphate status dependent. Cell 165, 464-474. doi: 10.1016/j.cell.2016.02.028

Hyde, K. D., Bussaban, B., Paulus, B., Crous, P. W., Lee, S., McKenzie, E. H. C., et al. (2007). Diversity of saprobic microfungi. Biodivers. Conserv. 16, 7-35. doi: 10.1007/s10531-006-9119-5

Kaul, S., Sharma, T., and, Dhar, M. K. (2016). "Omics" tools for better understanding the plant-endophyte interactions. Front. Plant Sci. 7:955. doi: $10.3389 /$ fpls.2016.00955

Kawahara, Y., Oono, Y., Kanamori, H., Matsumoto, T., Itoh, T., and Minami, E. (2012). Simultaneous RNA-seq analysis of a mixed transcriptome of rice and blast fungus interaction. PLoS ONE 7:e49423. doi: 10.1371/journal.pone. 0049423

Kelly, D. E., Kraševec, N., Mullins, J., and Nelson, D. R. (2009). The CYPome (Cytochrome P450 complement) of Aspergillus nidulans. Fungal Genet. Biol. 46, S53-S61. doi: 10.1016/j.fgb.2008.08.010

Lahrmann, U., Ding, Y., Banhara, A., Rath, M., Hajirezaei, M. R., Döhlemann, S., et al. (2013). Host-related metabolic cues affect colonization strategies of a root endophyte. Proc. Natl. Acad. Sci. U.S.A. 110, 13965-13970. doi: $10.1073 /$ pnas. 1301653110

Lahrmann, U., Strehmel, N., Langen, G., Frerigmann, H., Leson, L., Ding, Y., et al. (2015). Mutualistic root endophytism is not associated with the reduction of saprotrophic traits and requires a noncompromised plant innate immunity. New Phytol. 207, 841-857. doi: 10.1111/nph.13411

Maciá-Vicente, J. G., Jansson, H.-B., Talbot, N. J., and Lopez-Llorca, L. V. (2009). Real-time PCR quantification and live-cell imaging of endophytic colonization of barley (Hordeum vulgare) roots by Fusarium equiseti and Pochonia chlamydosporia. New Phytol. 182, 213-228. doi: $10.1111 / j .1469-8137.2008 .02743 . x$

Marcel, S., Sawers, R., Oakeley, E., Angliker, H., and Paszkowski, U. (2010). Tissueadapted invasion strategies of the rice blast fungus Magnaporthe oryzae. Plant Cell 22, 3177-3187. doi: 10.1105/tpc.110.078048

Martinez, D., Challacombe, J., Morgenstern, I., Hibbett, D., Schmoll, M., Kubicek, C. P., et al. (2009). Genome, transcriptome, and secretome analysis of wood decay fungus Postia placenta supports unique mechanisms of lignocellulose conversion. Proc. Natl. Acad. Sci. U.S.A. 106, 1954-1959. doi: 10.1073/pnas.0809575106

Mortazavi, A., Williams, B. A., McCue, K., Schaeffer, L., and Wold, B. (2008). Mapping and quantifying mammalian transcriptomes by RNA-Seq. Nat. Methods 5, 621-628. doi: 10.1038/nmeth.1226

Nawrot-Chorabik, K., Grad, B., Kowalski, T., and Woodward, S. (2016). Interactions between callus cultures of Pinus sylvestris and pine fungi with different trophic properties. For. Pathol. 46, 179-186. doi: 10.1111/efp.12240

O'connell, R. J., Thon, M. R., Hacquard, S., Amyotte, S. G., Kleemann, J., Torres, M. F., et al. (2012). Lifestyle transitions in plant pathogenic Colletotrichum fungi deciphered by genome and transcriptome analyses. Nat. Genet. 44, 1060-1065. doi: $10.1038 /$ ng.2372

Oliw, E. H., Aragó, M., Chen, Y., and Jernerén, F. (2016). A new class of fatty acid allene oxide formed by the dioxygenase-p450 fusion proteins of human and plant pathogenic fungi, Coccidioides immitis and Zymoseptoria tritici. J. Lipid Res. 57, 1518-1528. doi: 10.1194/jlr.M068981

Peters, S., and Schulz, B. (1998). Interactions in dual cultures of endophytic fungi with host and nonhost plant calli. Mycologia 90, 360-367. doi: 10.2307/3761393

Pohl, C. H., and Kock, J. L. (2014). Oxidized fatty acids as inter-kingdom signaling molecules. Molecules 19, 1273-1285. doi: 10.3390/molecules19011273

Porras-Alfaro, A., and Bayman, P. (2011). Hidden fungi, emergent properties: endophytes and microbiomes. Annu. Rev. Phytopathol. 49, 291-315. doi: 10.1146/annurev-phyto-080508-081831

Promputtha, I., Hyde, K. D., McKenzie, E. H. C., Peberdy, J. F., and Lumyong, S. (2010). Can leaf degrading enzymes provide evidence that endophytic fungi becoming saprobes? Fungal Divers. 41, 89-99. doi: 10.1007/s13225-0100024-6

Promputtha, I., Lumyong, S., Dhanasekaran, V., McKenzie, E. H., Hyde, K. D., and Jeewon, R. (2007). A phylogenetic evaluation of whether endophytes become saprotrophs at host senescence. Microb. Ecol. 53, 579-590. doi: 10.1007/s00248-006-9117-x

Rockman, M. V., and Kruglyak, L. (2006). Genetics of global gene expression. Nat. Rev. Genet. 7, 862-872. doi: 10.1038/nrg1964

Siddikee, M. A., Zereen, M. I., Li, C. F., and Dai, C. C. (2016). Endophytic fungus Phomopsis liquidambari and different doses of $\mathrm{N}$-fertilizer alter microbial community structure and function in rhizosphere of rice. Sci. Rep. 6:32270. doi: $10.1038 /$ srep 32270

Sieber, T. N., Siebercanavesi, F., and Dorworth, C. E. (1990). Simultaneous stimulation of endophytic Cryptodiaporthe hystrix and inhibition of acer macrophyllum callus in dual culture. Mycologia 82, 569-575. doi: $10.2307 / 3760047$

Su, Z. Z., Mao, L. J., Li, N., Feng, X. X., Yuan, Z. L., Wang, L. W., et al. (2013). Evidence for biotrophic lifestyle and biocontrol potential of dark septate endophyte Harpophora oryzae to rice blast disease. PLoS ONE 8:e61332. doi: 10.1371/journal.pone.0061332

Syed, K., and Yadav, J. S. (2012). P450 monooxygenases (P450ome) of the model white rot fungus Phanerochaete chrysosporium. Crit. Rev. Microbiol. 38, 339-363. doi: 10.3109/1040841X.2012.682050

Tsitsigiannis, D. I., and Keller, N. P. (2007). Oxylipins as developmental and host-fungal communication signals. Trends Microbiol. 15, 109-118. doi: 10.1016/j.tim.2007.01.005

Uranga, C. C., Beld, J., Mrse, A., Cordova-Guerrero, I., Burkart, M. D., and Hernández-Martínez, R. (2016). Fatty acid esters produced by Lasiodiplodia theobromae function as growth regulators in tobacco seedlings. Biochem. Biophys. Res. Commun. 472, 339-345. doi: 10.1016/j.bbrc.2016.02.104

Wang, H., Chen, H., Hao, G., Yang, B., Feng, Y., Wang, Y., et al. (2013). Role of the phenylalanine-hydroxylating system in aromatic substance degradation and lipid metabolism in the oleaginous fungus Mortierella alpina. Appl. Environ. Microbiol. 79, 3225-3233. doi: 10.1128/AEM.00238-13

Wang, H. W., Dai, C. C., Zhu, H., and Wang, X. X. (2014). Survival of a novel endophytic fungus Phomopsis liquidambari B3 in the indolecontaminated soil detected by real-time PCR and its effects on the indigenous microbial community. Microbiol. Res. 169, 881-887. doi: 10.1016/j.micres.2014. 05.006

Wang, H. W., Zhang, W., Su, C. L., Zhu, H., and Dai, C. C. (2015). Biodegradation of the phytoestrogen luteolin by the endophytic fungus Phomopsis liquidambari. Biodegradation 26, 197-210. doi: 10.1007/s10532-015-9727-4 
Weber, R. W., Stenger, E., Meffert, A., and Hahn, M. (2004). Brefeldin A production by Phoma medicaginis in dead pre-colonized plant tissue: a strategy for habitat conquest? Mycol. Res. 108, 662-671. doi: 10.1017/s0953756204000243

Wewer, V., Brands, M., and Dörmann, P. (2014). Fatty acid synthesis and lipid metabolism in the obligate biotrophic fungus Rhizophagus irregularis during mycorrhization of Lotus japonicus. Plant J. 79, 398-412. doi: 10.1111/tpj.12566

Xie, X. G., and Dai, C. C. (2015). Degradation of a model pollutant ferulic acid by the endophytic fungus Phomopsis liquidambari. Bioresour. Technol. 179, 35-42. doi: 10.1016/j.biortech.2014.11.112

Xu, X. H., Su, Z. Z., Wang, C., Kubicek, C. P., Feng, X. X., Mao, L. J., et al. (2014). The rice endophyte Harpophora oryzae genome reveals evolution from a pathogen to a mutualistic endophyte. Sci. Rep. 4:5783. doi: 10.1038/srep 05783

Yang, B., Ma, H. Y., Wang, X. M., Jia, Y., Hu, J., Li, X., et al. (2014a). Improvement of nitrogen accumulation and metabolism in rice (Oryza sativa L.) by the endophyte Phomopsis liquidambari. Plant Physiol. Biochem. 82, 172-182. doi: 10.1016/j.plaphy.2014.06.002

Yang, B., Wang, X.-M., Ma, H.-Y., Jia, Y., Li, X., and Dai, C.-C. (2014b). Effects of the fungal endophyte Phomopsis liquidambari on nitrogen uptake and metabolism in rice. Plant Growth Regul. 73, 165-179. doi: 10.1007/s10725-013-9878-4

Yang, B., Wang, X. M., Ma, H. Y., Yang, T., Jia, Y., Zhou, J., et al. (2015). Fungal endophyte Phomopsis liquidambari affects nitrogen transformation processes and related microorganisms in the rice rhizosphere. Front. Microbiol. 6:982. doi: $10.3389 /$ fmicb.2015.00982

Ye, J., Fang, L., Zheng, H., Zhang, Y., Chen, J., Zhang, Z., et al. (2006). WEGO: a web tool for plotting GO annotations. Nucleic Acids Res. 34, W293-W297. doi: $10.1093 /$ nar/gkl031
Yuan, J., Sun, K., Deng-Wang, M. Y., and Dai, C. C. (2016). The mechanism of ethylene signaling induced by Endophytic Fungus Gilmaniella sp. AL12 mediating sesquiterpenoids biosynthesis in Atractylodes lancea. Front. Plant Sci. 7:361. doi: $10.3389 /$ fpls.2016.00361

Zhou, J., Kang, L., Wang, H.-W., Yang, T., and Dai, C.-C. (2014a). Liquid laccase production by Phomopsis liquidambari B3 accelerated phenolic acids degradation in long-term cropping soil of peanut. Acta Agric. Scand. Sec. B - Soil Plant Sci. 64, 683-693. doi: 10.1080/09064710.2014. 953987

Zhou, J., Yang, T., Mei, Y.-Z., Kang, L., and Dai, C.-C. (2014b). Laccase production by Phomopsis liquidambari $\mathrm{B} 3$ cultured with food waste and wheat straw as the main nitrogen and carbon sources. J. Air Waste Manage. Assoc. 64, 1154-1163. doi: 10.1080/10962247.2014.930077

Zuccaro, A., Lahrmann, U., Guldener, U., Langen, G., Pfiffi, S., Biedenkopf, D., et al. (2011). Endophytic life strategies decoded by genome and transcriptome analyses of the mutualistic root symbiont Piriformospora indica. PLoS Pathog. 7:e1002290. doi: 10.1371/journal.ppat.1002290

Conflict of Interest Statement: The authors declare that the research was conducted in the absence of any commercial or financial relationships that could be construed as a potential conflict of interest.

Copyright (๑) 2017 Zhou, Li, Chen and Dai. This is an open-access article distributed under the terms of the Creative Commons Attribution License (CC BY). The use, distribution or reproduction in other forums is permitted, provided the original author(s) or licensor are credited and that the original publication in this journal is cited, in accordance with accepted academic practice. No use, distribution or reproduction is permitted which does not comply with these terms. 